\title{
Regulation of Neuronal Survival and Axon Growth by a Perinuclear cAMP Compartment
}

\author{
(i)Tomasz Boczek, ${ }^{1,2 \star}$ Evan G. Cameron, ${ }^{1 *}$ ๑Wendou Yu, ${ }^{3 \star}$ ๑Xin Xia, ${ }^{1}$ Sahil H. Shah, ${ }^{1}$ Boris Castillo Chabeco, ${ }^{3}$ \\ - Joana Galvao, ${ }^{1}$ Michael Nahmou, ${ }^{1}$ Jinliang Li, ${ }^{1,3}$ Hrishikesh Thakur, ${ }^{1,3}$ - Jeffrey L. Goldberg, ${ }^{1,3}$ \\ and $\oplus^{\oplus}$ Michael S. Kapiloff ${ }^{1,3,4}$ \\ ${ }^{1}$ Department of Ophthalmology, Byers Eye Institute, Mary M. and Sash A. Spencer Center for Vision Research, Stanford University School of Medicine, Palo \\ Alto, California 94034, ${ }^{2}$ Department of Molecular Neurochemistry, Medical University of Lodz, Poland 92215, ${ }^{3}$ Interdisciplinary Stem Cell Institute, \\ Leonard M. Miller School of Medicine, University of Miami, Miami, Florida, 33101, and ${ }^{4}$ Department of Medicine and Stanford Cardiovascular Institute, \\ Stanford University School of Medicine, Palo Alto, California 94034
}

cAMP signaling is known to be critical in neuronal survival and axon growth. Increasingly the subcellular compartmentation of cAMP signaling has been appreciated, but outside of dendritic synaptic regulation, few cAMP compartments have been defined in terms of molecular composition or function in neurons. Specificity in cAMP signaling is conferred in large part by A-kinase anchoring proteins (AKAPs) that localize protein kinase A and other signaling enzymes to discrete intracellular compartments. We now reveal that cAMP signaling within a perinuclear neuronal compartment organized by the large multivalent scaffold protein mAKAP $\alpha$ promotes neuronal survival and axon growth. mAKAP $\alpha$ signalosome function is explored using new molecular tools designed to specifically alter local cAMP levels as studied by live-cell FRET imaging. In addition, enhancement of mAKAP $\alpha$-associated cAMP signaling by isoform-specific displacement of bound phosphodiesterase is demonstrated to increase retinal ganglion cell survival in vivo in mice of both sexes following optic nerve crush injury. These findings define a novel neuronal compartment that confers cAMP regulation of neuroprotection and axon growth and that may be therapeutically targeted in disease.

Key words: cAMP; FRET imaging; neuroprotection; phosphodiesterase; signal transduction; signalosome

\section{Significance Statement}

cAMP is a second messenger responsible for the regulation of diverse cellular processes including neuronal neurite extension and survival following injury. Signal transduction by cAMP is highly compartmentalized in large part because of the formation of discrete, localized multimolecular signaling complexes by A-kinase anchoring proteins. Although the concept of cAMP compartmentation is well established, the function and identity of these compartments remain poorly understood in neurons. In this study, we provide evidence for a neuronal perinuclear cAMP compartment organized by the scaffold protein mAKAP $\alpha$ that is necessary and sufficient for the induction of neurite outgrowth in vitro and for the survival of retinal ganglion cells in vivo following optic nerve injury.

\section{Introduction}

Intracellular signal transduction is conveyed by second messengers that can act either by diffusion throughout the cell or within

Received Oct. 21, 2018; revised March 11, 2019; accepted April 10, 2019.

Author contributions: T.B., E.G.C., W.Y., J.L.G., and M.S.K. designed research; T.B., E.G.C., W.Y., X.X., B.C.C., J.G., M.N., J.L., and H.T. performed research; T.B., E.G.C., W.Y., S.H.S., B.C.C., J.G., J.L., and M.S.K. analyzed data; E.G.C., J.L.G., and M.S.K. wrote the paper.

This work was supported in part by National Institutes of Health Grants EY026766, EY022129, P30-EY026877 (M.S.K. and J.L.G.), EY025915 (E.G.C.), American Heart Association Grant 15SDG25700318 (W.Y.), and an unrestricted Grant from Research to Prevent Blindness.

The authors declare no competing financial interests.

*T.B., E.G.C., and W.Y. contributed equally as co-first authors.

Correspondence should be addressed to Michael S. Kapiloff at kapiloff@stanford.edu or Jeffrey L. Goldberg at jlgolbe@stanford.edu. discrete functional compartments to modulate diverse target effectors (Langeberg and Scott, 2015). The morphology of the neuron lends itself to compartmentalized signaling, given the extraordinarily large distances that often exist between axons, dendrites, and the soma, as well as because of the physical constraints upon diffusion conferred by the geometry of structures such as dendritic spines (Terenzio et al., 2017). For example, cAMP is an important second messenger for the development of nervous system connectivity, neuronal metabolism (Averaimo and Nicol, 2014; Stiles et al., 2014), growth cone guidance,

https://doi.org/10.1523/JNEUROSCI.2752-18.2019

Copyright $\odot 2019$ the authors 
A

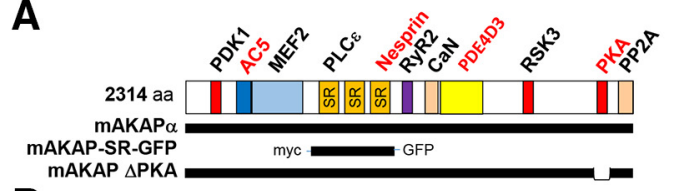

B

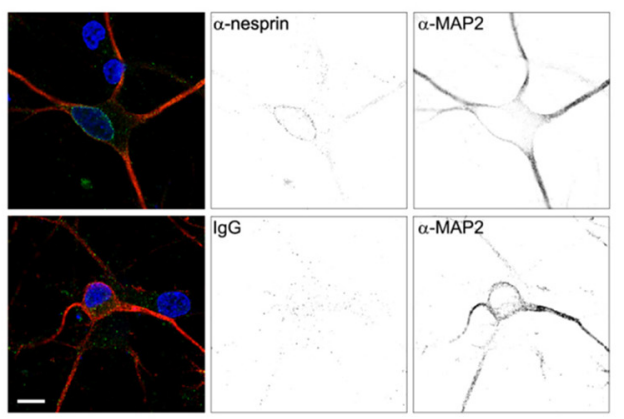

D

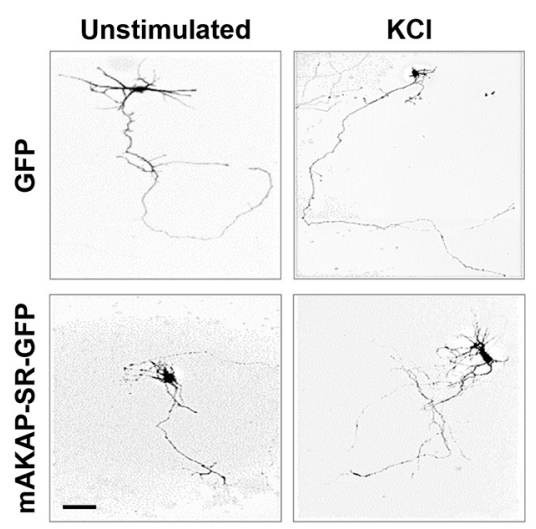

C

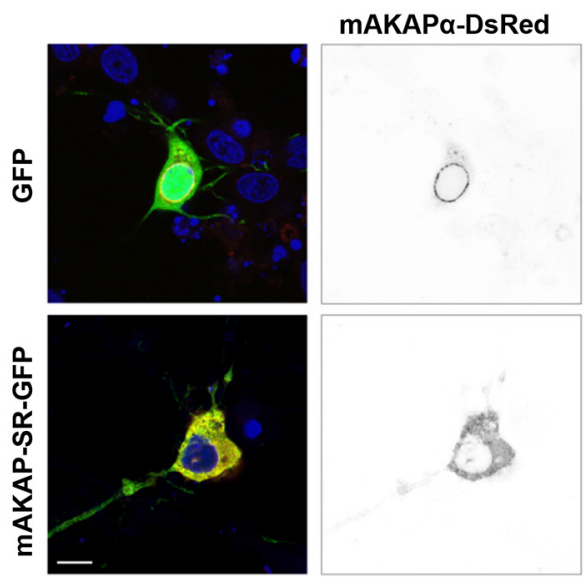

$\mathbf{E}$

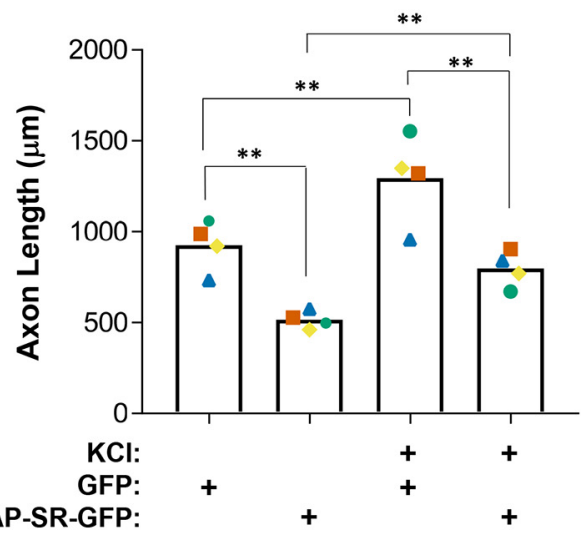

Figure 1. Perinuclear localization of mAKAP $\alpha$ at nesprin-1 $\alpha$ is required for primary hippocampal neuron neurite outgrowth. $A$, Structure of mAKAP $\alpha$ and expressed proteins. The three spectrin repeats (SRs) required for nuclear envelope targeting are indicated (Kapiloff et al., 1999). Binding sites are shown for those mAKAP binding partners for which there is evidence of direct binding: PDK1 (3-phosphoinositide-dependent kinase-1; Michel et al., 2005), AC5 (adenylyl cyclase 5; Kapiloff et al., 2009), MEF2 (Vargas et al., 2012), PLC $\varepsilon$ (phospholipase $(\varepsilon$; Zhang et al., 2011 ), nesprin-1 $\alpha$ (Pare et al., 2005a), RyR2 (ryanodine receptor; Marx et al., 2001), CaN (calcineurin; Li et al., 2010), PDE4D3 (Dodge et al., 2001), RSK3 (p90 ribosomal S6 kinase 3; Passariello et al., 2013), PKA (Kapiloff et al., 1999), and PP2A (protein phosphatase 2A; Dodge-Kafka et al., 2010). B. Hippocampal neurons stained with $\alpha$-nesprin (green) and $\alpha$-MAP2 antibodies (red) and DAPI nuclear stain (blue) with grayscale single channel images. C, Displacement of mAKAP by mAKAP-SR-GFP. Neurons expressing a mAKAP-DsRed fusion protein (red in composite and shown separately as grayscale image) and either GFP (green) or mAKAP-SR-GFP (green) and stained with DAPI (blue). Scale bar, $10 \mu \mathrm{m} . n=3$ for $\boldsymbol{B}$ and $\boldsymbol{C}$. $\boldsymbol{D}$, Neurons expressing mAKAP-SR-GFP or GFP control were cultured in defined media in the presence or absence of $40 \mathrm{~mm} \mathrm{KCl}$ for $2 \mathrm{~d}$. Grayscale images of GFP fluorescence are shown. Scale bar, $100 \mu \mathrm{m}$. E, Quantification of axon length. The length of the longest neurite was measured. Colors represent paired data for four independent experiments. ${ }^{* *} p \leq 0.01$.

activity-dependent neuronal survival and axon extension in vitro (Goldberg et al., 2002b), as well as neuroprotection and axonal regeneration in vivo (Wang et al., 2015). Despite being a watersoluble inherently diffusible second messenger, cAMP is subject to extensive compartmentation, especially with regard to the regulation of its canonical effector protein kinase A (PKA; Wild and Dell'Acqua, 2018). Localized to different cellular compartments by binding to A-kinase anchoring proteins (AKAPs), PKA is often found associated with specific adenylyl cyclase and phosphodiesterase (PDE) isoenzymes, such that cAMP fluxes can be locally modulated to regulate individual cellular processes. The functional significance of AKAP-mediated compartmentalization to neuronal function has been studied primarily in terms of synaptic transmission, most prominently cAMP signaling orchestrated by the postsynaptic scaffold protein AKAP79/150 relevant to synaptic plasticity and learning and memory (Wild and Dell'Acqua, 2018). Little is known, however, whether similar AKAP-associated microdomains play a role in other cAMPdependent neuronal functions.

mAKAP (AKAP6; Fig. 1A) is a modular scaffold protein localized to the nuclear envelope, which is expressed as a $250 \mathrm{kDa}$ alternatively-spliced $\alpha$-isoform in hippocampal neurons and retinal ganglion cells (RGCs) and a $230 \mathrm{kDa} \beta$-isoform in cardiac and skeletal myocytes (Michel et al., 2005; Pare et al., 2005a; Wang et al., 2015). mAKAP was initially identified as a PKA scaffold (Kapiloff et al., 1999) and was later found to bind both type 2 and type 5 adenylyl cyclase and the cAMP-specific PDE isoform PDE4D3, thereby providing the potential infrastructure for entirely local cAMP regulation within a perinuclear compartment (Dodge et al., 2001; Kapiloff et al., 2009). Additional research has revealed that $\mathrm{mAKAP}$ orchestrates large multimolecular signalosomes ( $>20$ binding partners identified) that transduce not only cAMP, but also calcium, phospholipid, mitogen-activated protein kinase, and hypoxic signaling (Passariello et al., 2015). Recently, we discovered that $\operatorname{mAKAP} \alpha$ expression is required for RGC survival and neurite growth in vitro and in vivo (Wang et al., 2015). The mechanisms by which $\operatorname{mAKAP} \alpha$ contributes to neuroprotection and neurite extension, however, remain unknown, including whether $\operatorname{mAKAP} \alpha$ dependent cAMP signaling is relevant to these processes. Using novel tools to specifically modulate local cAMP levels, we now demonstrate that cAMP in the perinuclear $\mathrm{mAKAP} \alpha$ compart- 
Table 1. Antibodies used in this study

\begin{tabular}{lll}
\hline Antigen & Species, catalog \# & Company \\
\hline $\begin{array}{l}\text { Blll-tubulin } \\
\text { GFP }\end{array}$ & Mouse, E7 monoclonal & Developmental Studies Hybridoma Bank \\
GFP & Rabbit, sc-8334 & Santa Cruz Biotechnology \\
Flag tag & Chicken, ab13970 & Abcam \\
HA tag & Rabbit, F7425 & Sigma-Aldrich \\
Myctag & Mouse, HA-7 monoclonal & Sigma-Aldrich \\
$\begin{array}{l}\text { Myctag } \\
\text { nesprin-1 }\end{array}$ & Mouse, 4A6 monoclonal & Millipore \\
mAKAP & Rabbit, 06-549 & Millipore \\
MAP2 & Mouse, MANNES1A (7A12) & Invitrogen \\
RBPMS & V054 & Kapilofflaboratory (Kapiloff et al., 1999; Pare et al., 2005a) \\
\hline
\end{tabular}

ment is necessary and sufficient for neurite extension in vitro. Further, we show that the mAKAP $\alpha$ compartment is defined, in part, by PDE4D3, which modulates local cAMP fluxes. Last, we demonstrate the potential of PDE4D3 anchoring disruption as a therapeutic strategy to promote neuroprotection after injury in vivo.

\section{Materials and Methods}

Plasmid constructs. A description of relevant plasmids and viruses is in the following paragraphs of this section. Additional details and complete vector maps for all constructions are available upon request. Many of these plasmids were constructed by GENEWIZ using methods of the company's choice. Plasmid constructs were validated by sequencing and by expression of the encoded recombinant proteins in Cos-7 cells. The "pS" series of vectors in which the conditional tetracycline-responsive promoter has been replaced with the CMV immediate early promoter are adenoviral shuttle vectors based upon the pTRE vector (Clontech) containing I-Ceu I and PI-Sce I sites for subcloning into the adenovirus bacterial vector Adeno-X (Clontech). Adenovirus was purified after amplification using Vivapure AdenoPACK kits (Sartorius) and titered using HEK293 cells. AAV were produced by the University of Pennsylvania Vector Core with funding provided in part by the National Heart, Lung, and Blood Institute Gene Therapy Resource Program.

pS-mCherry-PDE4D_C (ERK-)-nesprin expression plasmid includes a cDNA expressing the following polypeptides: mCherry-human PDE4D3 catalytic domain (aa 225-673; NP_006194.2) with missense mutations K455A/K456A/S579A/F597A/Q598A/F599A-myc tag-human nesprin-1 $\alpha$ (AAN60442.1; aa 7799-8797). pS-mCherry-AC-nesprin contains a fragment of the rat soluble adenylyl cyclase cDNA encoding the $\mathrm{C} 1+\mathrm{C} 2$ catalytic domains (NP067716.1; aa 1-469) inserted in place of the PDE BsrGI-NotI region of pS-mCherry-PDE4D_C (ERK-)nesprin. pS-mCherry-nesprin control vector is the same as the above vectors except lacking the EcoRI-XhoI fragment containing the AC or PDE domain and myc-tag sequences.

pscS2-4D3(E)-mCherry-mh is a shuttle vector for both subcloning into adenovirus and for directly producing self-complementary AAV2sc.4D3(E), containing the following: (1) AAV2 (NC_001401.2), bp 4664-4489 in antisense orientation 5' to a PI-Sce I site; (2) CMV immediate early promoter; (3) a cDNA expressing human PDE4D3 aa residues $1-20$ with a S13E mutation, (ELAAK) ${ }_{3}$ flexible linker-mCherry-myc tag$\mathrm{His}_{6}$ tag fusion protein; (4) SV40 poly A sequence; and (5) AAV2 (NC_001401.2), bp 4559-4662 3' to a I-Ceu I site. pscS2-mCherry-mh control vector was constructed by deleting in pscS2-4D3(E)mCherry-mh a NheI-Age I fragment that encodes the 4D3(E) peptide.

The FRET-based PKA sensor AKAR4 in pcDNA3 was kindly provided by Dr. Jin Zhang (Johns Hopkins University; Depry et al., 2011). pSAKAR4 adenoviral shuttle vector was constructed by subcloning the AKAR4 cDNA from pCDNA3-AKAR4 into the NheI and PspOMI sites of $\mathrm{pS}$-mCherry-Nesprin. The shuttle vector $\mathrm{pS}$-AKAR4-Nesprin $1 \alpha$ encodes perinuclear-AKAR4 (PN-AKAR4), which includes human nesprin-1 $\alpha$ (AAN60442.1; aa 7799-8797) at the C-terminus of AKAR4.

Plasmids and adenovirus for rat mAKAP and control shRNA and encoding myc-tagged mAKAP 586-1286 (myc-mAKAP-SR) were as previously described (Pare et al., 2005a,b). Adenovirus expressing $\mathrm{N}$-terminally myc-tagged rat $\mathrm{mAKAP} \alpha$ were generated using a pTRE (Clontech) expression vector containing a cDNA with a myc-tag followed by a full-length mAKAP $\alpha$ open reading frame (NM_022618.1; bp 128-7138). mAKAP $\alpha \Delta$ PKA was expressed using adenovirus containing a deletion of mAKAP bp 6284-6346 (codons 2053-2073). Expression vector pEGFP-PDE4D3-vsv was as previously described (Dodge-Kafka et al., 2010).

The antibodies used in this study are listed in Table 1.

Animal models. All in vivo research was performed under the supervision of the Institutional Animal Care and Use Committee at the University of Miami or Stanford University. All rats used in this project were Sprague-Dawley, and all mice used in this project were C57BL/6.

\section{Cell culture}

Cos -7 cells. Cos- 7 cells were maintained in DMEM $(10 \% \mathrm{v} / \mathrm{v}$ FBS $)$ at $37^{\circ} \mathrm{C}$ in a humidified incubator with $5 \% \mathrm{CO}_{2}$. For live cell imaging, Cos-7 cells were plated onto 25-mm-diameter sterilized glass coverslips in 6-well plates and were either transfected with polyethylenimine or infected with adenovirus at $60-70 \%$ confluence and allowed to grow for $24-48 \mathrm{~h}$ before live cell imaging. Nesprin- $1 \alpha$ fusion proteins are not properly localized to the nuclear envelope when grossly overexpressed due to saturation of Klarsicht/ANC-1/Syne-1 homology (KASH)-SUN domain protein-protein interactions (Zhou et al., 2018). Only Cos-7 cells and neurons with epifluorescence for PN-AKAR and the other nesprin- $1 \alpha$ fusion proteins restricted to the nuclear envelope were included in the studies.

Primary rat hippocampal neurons. Hippocampal cultures were prepared from Sprague-Dawley rat embryonic day 18 embryos. Briefly, the rat hippocampal CA1-CA3 region was dissected in PBS medium with 10 mM D-glucose and digested with $0.05 \%$ trypsin-EDTA in PBS with $11 \mathrm{~mm}$ D-glucose for $30 \mathrm{~min}$ at $37^{\circ} \mathrm{C}$. The dissociated tissues were centrifuged at $250 \times g$ for 2 min and then triturated with fire polished glass pipet in HBSS with calcium and magnesium in plating medium (10\% v/v horse serum in DMEM). Dissociated neurons were plated on nitric acidtreated $25 \mathrm{~mm}$ cover glass coated with poly-L-lysine in plating medium. Four hours after plating, the medium was replaced with maintenance medium supplemented with $1 \% \mathrm{~N} 2,2 \%$ B27 (Invitrogen), $5 \mathrm{~mm}$ D-glucose, $1 \mathrm{~mm}$ sodium pyruvate. Four days later, $4 \mu \mathrm{M}$ arabinosyl cytosine was added to inhibit glial proliferation, and the neurons were either transfected with JPEI or infected with adenovirus.

Live cell imaging was performed $36-72 \mathrm{~h}$ after transfection/infection as described in Live cell FRET imaging. For neurite extension assays, the cells were cultured for $2 \mathrm{~d}$ in DMEM with $1 \mu \mathrm{g} / \mathrm{ml}$ chicken egg albumin and $1 \mathrm{~mm}$ sodium pyruvate. Forty millimolar $\mathrm{KCl}, 10 \mu \mathrm{M}$ forskolin (FSK), $100 \mu \mathrm{M}$ 3-isobutyl-1-methylxanthine (IBMX), $20 \mu \mathrm{M}$ Milrinone, and $10 \mu \mathrm{M}$ Rolipram were included as indicated. Two days later, the neurons were fixed and stained with antibodies. Nuclei were counterstained with DAPI and SlowFade Gold antifade solution (Invitrogen) was added before coverslip mounting. Images were acquired with IPLab or SlideBook 6 by wide-field microscopy (Leica DMI 6000B or Zeiss Axio Observer 7) and processed with Adobe Photoshop CSS 12.1. The length of the longest neurite for $\sim 15$ neurons average per condition was measured for each experiment with ImageJ with Simple Neurite Tracer pl- 
ugin. Other neuronal images were acquired using a Zeiss 880 confocal microscope.

Retinal ganglion cells. RGCs were purified (N99.5\%) from postnatal (P2-P4) Sprague-Dawley rats through sequential immunopanning, as previously described (Goldberg et al., 2002b). Following purification, RGCs were seeded at $1000-2500$ cells/well in poly-D-lysine- $(70 \mathrm{kDa}, 10$ $\mu \mathrm{g} / \mathrm{ml}$; Sigma-Aldrich) and laminin- (1 $\mu \mathrm{g} / \mathrm{ml}$; Invitrogen) coated $24-$ well plates. RGCs were cultured in neurobasal serum-free defined medium containing pen/strep $(100 \times)$, insulin $(5 \mu \mathrm{g} / \mathrm{ml})$, sodium pyruvate (1 mM), L-glutamine (1 mM), triiodothyronine $(40 \mathrm{ng} / \mathrm{ml}$; SigmaAldrich), $N$-acetyl cysteine (5 $\mu \mathrm{g} / \mathrm{ml}$; Sigma-Aldrich), B27 (1:50), brainderived neurotrophic factor (BDNF; $50 \mathrm{ng} / \mathrm{ml}$ ), ciliary neurotrophic factor $(10 \mathrm{ng} / \mathrm{ml})$, and FSK $(5 \mu \mathrm{M})$ as described previously (MeyerFranke et al., 1995). Four hours after seeding, RGCs were incubated with AAV2-4D3-mCherry or -mCherry viral particles at 75,000 MOI for $20 \mathrm{~h}$ followed by a half media change the next day. After an additional $3 \mathrm{~d}$ incubation in $10 \% \mathrm{CO}_{2}$ at $37^{\circ} \mathrm{C}$, the RGCs were fixed and stained for $\beta$ III-tubulin. Images were acquired on a Zeiss Axio Observer inverted microscope and the longest neurite per cell ( $\sim 15$ cells average per condition per experiment) measured using ImageJ Neurite Tracer. Dead, overlapping, and mCherry-negative RGCs were excluded from the analysis.

Live cell FRET imaging. Live cell images were acquired using either (1) a DMI6000B inverted microscope (Leica) with $63 \times$ Plan Apo/1.25 HCX PL FLUOTAR objective, and LB10-NWIQ component (fluorescent light source, filter wheel, ultrafast shutter, Leica) and Qimaging Retiga EXi camera driven by SlideBook 6.0, or (2) automated, inverted Zeiss Axio Observer 7 Marianas Microscope equipped with a X-Cite 120LED Boost White Light LED System and a high-resolution Prime Scientific CMOS digital camera that is controlled by a workstation loaded with SlideBook imaging and microscope control software (Intelligent Imaging Innovations). Filters were as follows: Dichroic: FF459/526/596-Di01; CFP Exciter: FF02-438/24; CFP Emitter: FF01-482/25; YFP Exciter: FF01-509/ 22; YFP Emitter: FF01-544/24; mCherry Exciter: FF01-578/21; mCherry Emitter: FF02-641/75. Cells were washed twice before imaging in PBS with $11 \mathrm{~mm}$ D-glucose and perfused during imaging with Tyrode solution (in mM: $137 \mathrm{NaCl}, 2.7 \mathrm{KCl}, 1 \mathrm{MgCl}_{2}, 2 \mathrm{CaCl}_{2}, 0.2 \mathrm{Na}_{2} \mathrm{HPO}_{4}, 12 \mathrm{NaHCO}_{3}$, $11 \mathrm{D}$-glucose, $25 \mathrm{HEPES}, 1 \% \mathrm{BSA})$ at room temperature $\left(23-25^{\circ} \mathrm{C}\right)$ in a perfusion chamber (Warner Instruments). For stimulation of cells, the bath solution was exchanged by peristaltic pump (Harvard Apparatus) perfusion with different drugs in Tyrode solution. During live cell imaging the exposure time for FRET, acceptor, and donor channels was 100 $\mathrm{ms}$ and images were collected every $10 \mathrm{~s}$. Baseline images were acquired for 2-5 min. All imaging processing was performed using SlideBook software. Net FRET for regions of interest was calculated by subtracting bleedthrough for both the donor and acceptor channels after background-subtraction. FRET ratio " $R$ " was net FRET $\div$ backgroundsubtracted donor signal, with $R_{0}$ being the ratio for time $=0$.

Intravitreal injection and optic nerve crush. AAV2-4D3-mCherry or -mCherry control $\left(2 \mu \mathrm{l} 5-7 \times 10^{12} \mathrm{vg} / \mathrm{ml}\right)$ was injected intravitreally into adult P20-P30 wild-type mice of either sex 2 weeks before optic nerve crush. Intravitreal injections were performed just posterior to the pars plana with a 31-gauge needle (Hamilton) connected to a $5 \mu$ l Hamilton syringe. Care was taken not to damage the lens. For nerve crush, the left optic nerve was exposed from the outer canthus and crushed for $5 \mathrm{~s}$ with a Dumont \#5 forceps (91150-20, FST) $\sim 1.5 \mathrm{~mm}$ behind the globe. Care was taken to avoid damaging the blood supply to the retina. Mice with any significant postoperative complications (e.g., retinal ischemia, cataract) were excluded from further analysis.

Two weeks after optic nerve crush, mice were killed by intracardial perfusion with $4 \%$ PFA. Retinal flat-mount was prepared as described previously (Zhao et al., 2014). Briefly, the eyes were removed and postfixed with $4 \%$ PFA for $2 \mathrm{~h}$ at room temperature. Retinas were flat mounted in mounting medium (ProLong Gold Anti-Fade, ThermoFisher Scientific) on glass slides and stained with RNA binding protein with multiple splicing (RBPMS) antibodies. Confocal images were acquired with a confocal laser scanning microscope (Zeiss 880 ) and a $\times 10$ magnification lens. The imaging and quantification were performed in a masked fashion as previously described (Wang et al., 2015). Briefly, the retinas were divided into four quadrants, and one digital micrograph was taken from a fixed distance from the periphery of each of the four fields. Although mCherry epifluorescence was not evenly distributed throughout the retina (data not shown), RBPMS-positive RGCs were counted regardless of the apparent level of AAV-based expression.

Statistics. Statistics were computed using GraphPad Prism 7. All unpaired data are expressed as mean \pm SEM. Error bars are not provided for bar graphs showing paired experiments or scatter plots. Repeated symbols are used as follows: ${ }^{*} p \leq 0.05,{ }^{* *} p \leq 0.01,{ }^{* * *} p \leq 0.001$. Single comparisons were by two-tailed Student's $t$ tests, paired or unpaired as appropriate. All datasets involving multiple comparisons for which $p$ values are provided were significant by ANOVA, $\alpha=0.05$. One-way or two-way ANOVA were performed with matching as appropriate. $p$ values for experiments involving multiple comparisons were obtained by Tukey post hoc testing, albeit $p$ values for not all comparisons are indicated on the graphs.

\section{Results}

mAKAP $\alpha$ anchoring by nesprin- $1 \alpha$ is required for neurite extension in vitro

We have demonstrated that $\operatorname{mAKAP} \alpha$ expression is required for neurite growth in vitro and neuronal survival in vivo (Wang et al., 2015), but how its perinuclear localization affects these processes has not been explored. mAKAP is localized to the nuclear envelope via protein-protein interactions with $\mathrm{KASH}$ domain-containing, transmembrane isoforms of nesprin-1 that heterodimerize via their spectrin-repeat domains with mAKAP (Pare et al., 2005a). Importantly, the short nesprin-1 $\alpha$ isoform sufficient to direct mAKAP to the nuclear envelope is highly expressed in myocytes and RGCs of the adult retina (Pare et al., 2005a; Razafsky et al., 2013). We now show that nesprin-1 is present on the nuclear envelope in hippocampal neurons (Fig. $1 B$ ), just as we have previously shown for mAKAP $\alpha$ (Michel et al., 2005) and as found for nesprin-1 in other neurons and other cell types (Gros-Louis et al., 2007; Zhou et al., 2018). Expression of a GFP-tagged fragment encoding the mAKAP spectrin-repeat domains (amino acid residues 586-1286, "mAKAP-SR-GFP"; Fig. $1 A$ ) will displace mAKAP $\beta$ from nesprin- $1 \alpha$ at the nuclear envelope in myocytes (Pare et al., 2005a). Expression of mAKAP-SRGFP similarly displaced DsRed-tagged $\operatorname{mAKAP} \alpha$ from the nuclear envelope in neurons (Fig. 1C), implying that the mechanism for mAKAP $\alpha$ localization is conserved in these cells.

Following our previous publication demonstrating that mAKAP $\alpha$ expression is required for axon growth (Wang et al., 2015), we tested whether proper $\operatorname{mAKAP} \alpha$ localization was also required for axon growth. Hippocampal neurons were infected with adenovirus expressing the delocalizing mAKAP-SR-GFP peptide in the presence or absence of $\mathrm{KCl}$ that induces activitydependent neurite extension via cAMP and PKA-dependent mechanisms (Goldberg et al., 2002b). Outgrowth measurements were performed following $3-5 \mathrm{~d}$ in vitro. By this time in culture, both hippocampal (and RGC) neurons are typically polarized with the longest neurite being the elongating axon (Gorshkov et al., 2017). Expression of mAKAP-SR-GFP inhibited axon outgrowth as assayed by measurement of longest neurite length, both for neurons cultured in defined media, as well as neurons stimulated by chronic $\mathrm{KCl}$ depolarization (Fig. $1 D, E$ ). These results suggest that proper localization of the mAKAP $\alpha$ scaffold, and not merely expression (as shown previously in RGCs; Wang et al., 2015), is critical for axon extension. These data also support the premise for investigating whether perinuclear cAMP at $\operatorname{mAKAP} \alpha$ signalosomes has a unique function in neurons.

\section{Generation of an mAKAP $\alpha$-dependent PKA activity sensor}

PKA activity in living cells can be assayed with spatiotemporal resolution using genetically encoded FRET biosensors (Zhou et 
A

B
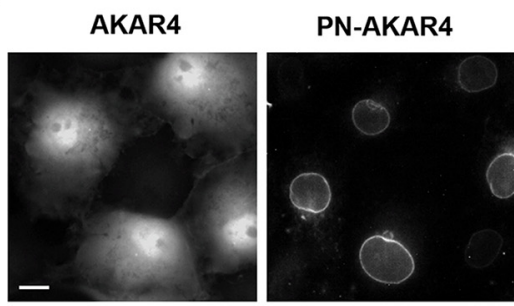

D

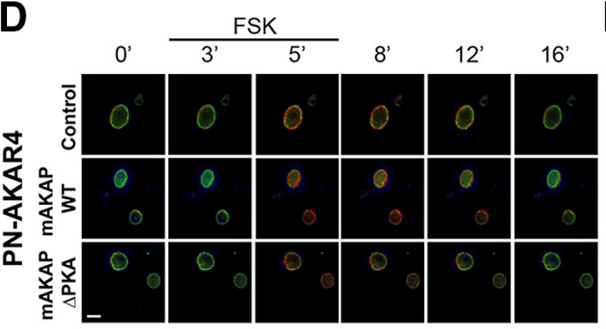

F

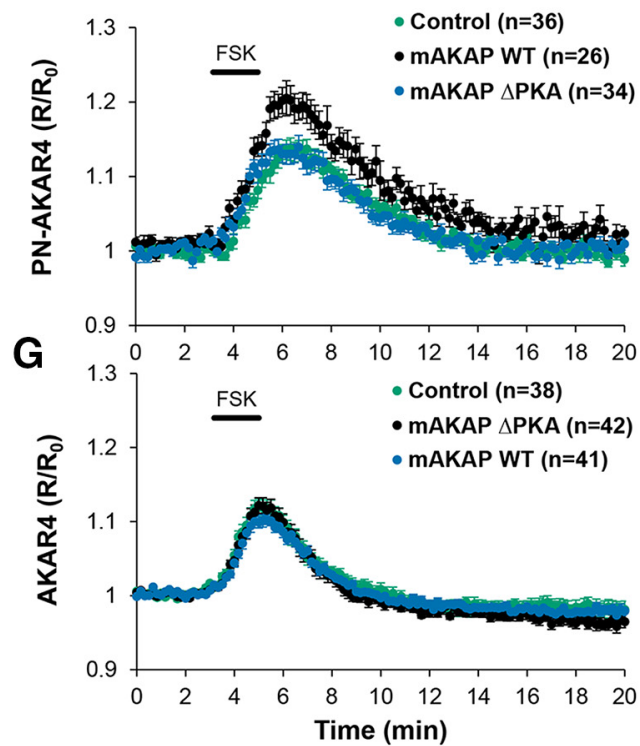

E

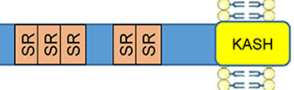

C
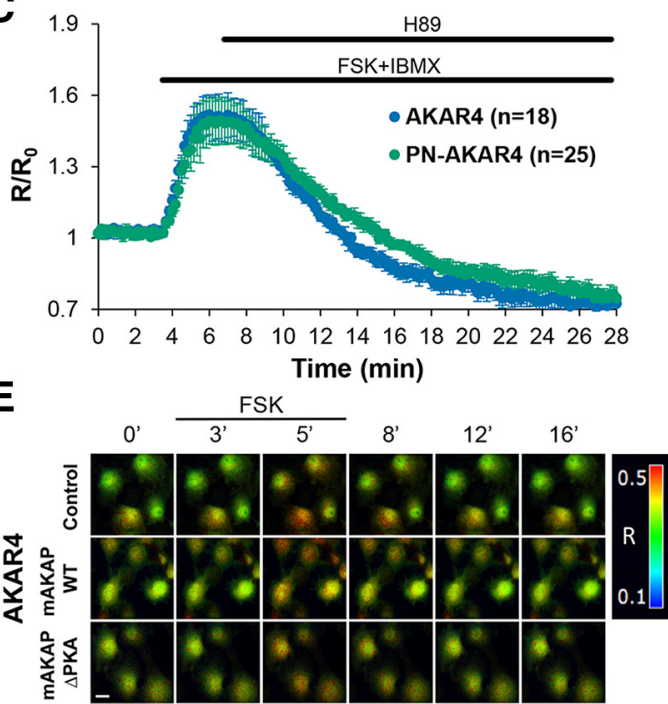

Amplitude $\left(\mathbf{R} / \mathbf{R}_{0}\right)$

$t_{1 / 2}(s)$
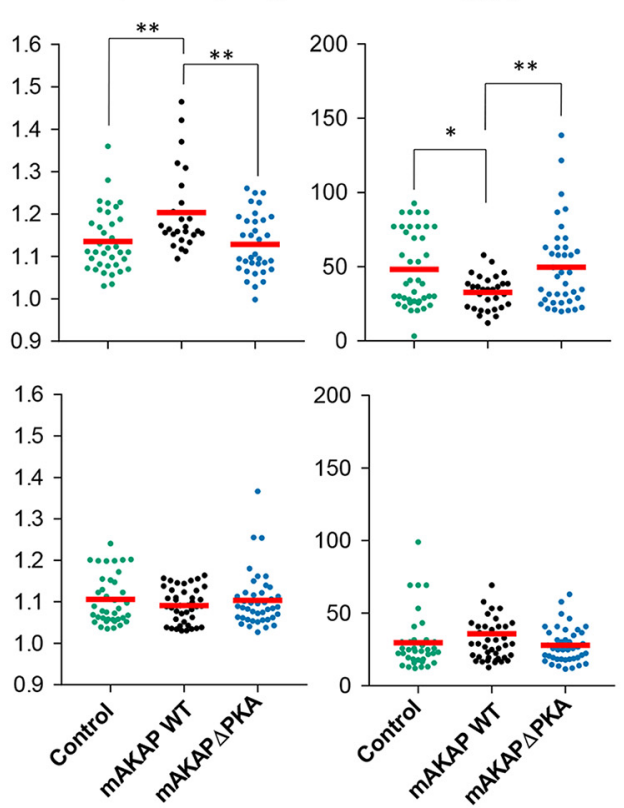

Figure 2. Characterization of a new perinuclear PKA FRET sensor. A, AKAR4 is a FRET biosensor that exhibits increased signal upon phosphorylation of the LRRATLVD PKA peptide substrate (Depry et al., 2011). PN-AKAR4 is an AKAR4-nesprin- $1 \alpha$ fusion protein. Nesprin- $1 \alpha$ (dark blue) contains 5 spectrin repeats (SRs) and a transmembrane KASH domain that localizes the protein to the nuclear envelope. $\boldsymbol{B}$, Grayscale CFP images of Cos-7 cells expressing AKAR4 or PN-AKAR4. Scale bar, $10 \mu \mathrm{m}$. C, Average normalized FRET ratio signal \pm SEM. (R/R) following stimulation with $10 \mu \mathrm{m}$ FSK and $100 \mu \mathrm{m}$ IBMX and then inhibition with $10 \mu \mathrm{m}$ H89. D, E, Pseudocolored images showing FRET ratio $R$ (net FRET $\div$ background-subtracted donor signal) for representative tracings for $\boldsymbol{F}$ and $\mathbf{G}$. Color hue and not intensity is indicative of $R$, as shown on the color scale. $F, G$, Cos-7 cells expressing sensor and either mAKAP $\alpha$ WT or PKA binding mutant (mAKAP $\triangle$ PKA) were stimulated with $10 \mu \mathrm{m}$ FSK for $2 \mathrm{~min}$ (bar on graph). Average tracings $\left(R / R_{0} \pm S E M\right)$ and the peak amplitude and half-time of signal decay $\left(t_{1 / 2}\right)$ for individual tracings are shown; red bars indicate mean. ${ }^{*} p \leq 0.05,{ }^{* *} p \leq$ 0.01 .

al., 2012). AKAR4 is a well characterized biosensor that contains a PKA target site and a FH1 phospho-amino acid-binding domain inserted between donor cerulean and acceptor cpVenusE172 fluorescent proteins (Fig. $2 A$ ), such that sensor phosphorylation increases FRET signal (Depry et al., 2011; Gorshkov et al., 2017). To assay PKA activity in a mAKAP-specific perinuclear compartment, AKAR4 was expressed in fusion to the $\mathrm{N}$-terminus of nesprin- $1 \alpha$ (Fig. $2 A$ ). Initial characterization of PN-AKAR4 was performed in Cos-7 cells, a heterologous cell line that lacks nesprin- $1 \alpha$ and mAKAP. When the fusion biosensor was expressed at moderate levels to avoid saturation of the KASH domain-mediated nuclear envelope localization mechanism (Zhou et al., 2018), cerulean and cpVE172-E172 fluorescence were limited to the nuclear envelope (Fig. 2B). Maximum and minimum FRET signals were obtained for both PN-AKAR4 and AKAR4 by infusing the adenylyl cyclase activator FSK and the 
PDE inhibitor IBMX followed by the PKA inhibitor H-89, demonstrating that the localized sensor had the same dynamic range of response as the diffusely localized parent sensor (Fig. 2C). Likewise, a 2 min pulse of FSK resulted in a similar transient increase in PN-AKAR4 FRET signal as AKAR4 sensor (Figs. 2D$G)$. Coexpression of $\mathrm{mAKAP} \alpha$ resulted in a PN-AKAR4 transient that was $\sim 50 \%$ greater in amplitude and exhibited a more rapid signal decay $\left(t_{1 / 2}\right)$, consistent with the recruitment by mAKAP $\alpha$ of both PKA (increased amplitude) and PDE4D3 (decreased $t_{1 / 2}$ ) to the nesprin- $1 \alpha$ perinuclear compartment. A full-length mAKAP $\alpha$ PKA-binding mutant lacking PKA-binding residues 2053-2073 (Pare et al., 2005b; mAKAP $\alpha \Delta$ PKA; Fig. 1A) did not enhance PN-AKAR4 amplitude nor reduce $t_{1 / 2}$, consistent with PDE4D3 being a PKA-activated PDE (Dodge et al., 2001). In addition, coexpression of mAKAP $\alpha$ WT (and mAKAP $\alpha \Delta$ PKA) did not significantly affect the level of PKA activity detected by the parent sensor (Fig. 2G). Together these data show that nesprin- $1 \alpha$ fusion results in a PKA biosensor that can detect $\operatorname{mAKAP} \alpha-$ dependent signals localized to a perinuclear compartment.

RNA interference of endogenous $\operatorname{mAKAP} \alpha$ expression revealed that PN-AKAR4 is a mAKAP $\alpha$-dependent biosensor in hippocampal neurons, where the sensor was similarly localized to the nuclear envelope (Fig. $3 A, B$ ). FSK stimulation of PN-AKAR4 in neurons resulted in a PKA transient whose amplitude was inhibited $\sim 75 \%$ by coexpression of a mAKAP shRNA (Pare et al., 2005b) and whose signal decay was twofold slower (Figs. 3C,E). Importantly, mAKAP $\alpha$ depletion had no significant effect upon signal detected by the parent AKAR4 present in the soma or the neurites of the neurons (Fig. $3 D, F, G$ ). Together with the data obtained in heterologous cells, these results show that PNAKAR4 is a reporter specific for PKA activity associated with $\operatorname{mAKAP} \alpha$ signalosomes at the neuronal nuclear envelope, where mAKAP $\alpha$ signalosome formation affects the kinetics and amplitude of PKA signaling.

\section{Increased cAMP at mAKAP $\alpha$ promotes neurite extension}

FSK stimulation to globally elevate cAMP levels induces axon growth in neurons, and blocking mAKAP expression decreases axon growth in vitro (Wang et al., 2015), raising the hypothesis that CAMP/PKA activity in the mAKAP perinuclear compartment promotes axon growth. To determine whether elevating cAMP specifically at $\mathrm{mAKAP} \alpha$ signalosomes is sufficient to induce neurite outgrowth, we constructed an mCherry-tagged protein containing the constitutively-active adenylyl cyclase catalytic domain from soluble adenylyl cyclase (ADCY10) fused to nesprin- $1 \alpha$ to locally synthesize cAMP in that compartment (mCherry-AC-nesprin; Fig. 4A). Transient coexpression of mCherry-AC-nesprin with PN-AKAR4 (Fig. 4B) resulted in a 2.7-fold increased baseline FRET signal compared with mCherry-nesprin control (Fig. 4C). Importantly, expression of mCherry-AC-nesprin had no effect on parent AKAR4 in soma or neurites, showing that the constitutively increased cAMP production was limited to the $\operatorname{mAKAP} \alpha /$ nesprin- $1 \alpha$ perinuclear compartment (Fig. 4C). With this tool to specifically increase cAMP/PKA activity in the perinuclear compartment, we turned to its effect on axon growth. Measurement of the longest neurite per cell revealed that expression of mCherry-AC-nesprin increased axon extension compared with neurons expressing control mCherry-nesprin (Fig. 5; 32\% and 31\% in the absence and presence of $\mathrm{KCl}$, respectively). These results imply that cAMP signaling spatially restricted to the $\operatorname{mAKAP} \alpha$ perinuclear compartment is sufficient to induce hippocampal neuron axon outgrowth.

\section{Increased PDE activity at mAKAP $\alpha$ suppresses neurite extension}

We next asked the converse question, whether cAMP elevation at the $\mathrm{mAKAP} \alpha /$ nesprin- $1 \alpha$ perinuclear compartment is necessary for neurite growth. To prevent cAMP signaling at $\operatorname{mAKAP} \alpha$ signalosomes, an mCherry-tagged protein was constructed containing the catalytic domain of PDE4D fused to nesprin- $1 \alpha$ (mCherry-PDE-nesprin; Fig. 6A,B) to constitutively degrade cAMP near the mAKAP $\alpha$ scaffold. ERK mitogen-activated protein kinase (MAP-kinase) can bind and phosphorylate PDE4D, resulting in PDE4D inhibition (Klussmann, 2016). To preclude inhibition of the PDE construct by ERK signaling, the KIM and FQF docking sites and Ser-579 phosphorylation site on the PDE4D catalytic domain (PDE*; Fig. 6A) were ablated by missense mutation (MacKenzie et al., 2000). Transient coexpression of mCherry-PDE-nesprin reduced baseline PN-AKAR4 FRET signal $36 \%$ in hippocampal neurons compared with cells expressing control mCherry-nesprin (Fig. 6C,E). In addition, expression of mCherry-PDE-nesprin completely prevented the induction of a PN-AKAR4 FRET transient by FSK in hippocampal neurons (Figs. 6C,F). Importantly, increased perinuclear PDE activity had no effect on AKAR4 FRET signals either in the soma or neurites (Fig. 6D,G-J), demonstrating compartment-specific cAMP depletion.

Consistent with decreased perinuclear cAMP signaling in neurons expressing mCherry-PDE-nesprin, axon growth was significantly inhibited $21 \%$ compared with cells expressing control mCherry-nesprin (Fig. 7). Importantly, chronic $\mathrm{KCl}$ depolarization was unable to induce axon extension in cells expressing the mCherry-PDE-nesprin construct indicating that enhanced PDE activity at mAKAP $\alpha /$ nesprin- $1 \alpha$ complexes can suppress depolarization-induced growth (Fig. $7 B$, bars 3 and $6 ; p=0.95$ for mCherry-PDE-nesprin $\pm \mathrm{KCl}$ ). Together with the above results, these data show that cAMP signaling at perinuclear $\operatorname{mAKAP} \alpha$ signalosomes is both sufficient and necessary for neurite extension in cultured hippocampal neurons.

\section{mAKAP $\alpha$-associated PDE4D3 regulates neurite extension}

Given our new findings that $\operatorname{mAKAP} \alpha$-associated perinuclear cAMP regulates neurite extension, we asked whether inhibition of endogenous $\mathrm{mAKAP} \alpha$-associated PDE activity would similarly promote neurite outgrowth. The mAKAP $\alpha$ scaffold binds the type 4 cAMP-specific phosphodiesterase PDE4D3 (Dodge et al., 2001). First, we tested whether inhibition using the PDE4 inhibitor rolipram would promote neurite extension in hippocampal neurons. Similar to results previously obtained for motoneurons (Aglah et al., 2008), addition of rolipram to the culture medium induced neurite extension comparably to that with $\mathrm{KCl}$ depolarization to induce adenylyl cyclase activity via calciumdependent signaling (Goldberg et al., 2002b), FSK to induce adenylyl cyclase activity directly, and IBMX to inhibit all PDEs (Fig. 8 ). Consistent with the recognized differences between PDE3 and PDE4 activity in neurons (Gorshkov et al., 2017), PDE3 inhibition with milrinone had no effect on neurite growth.

To specifically target and disrupt PDE4D3-mAKAP binding, we took advantage of the fact that the 4D3-specific N-terminal peptide confers direct binding to mAKAP within residues $1286-$ 1401 (Dodge et al., 2001; Carlisle Michel et al., 2004). We generated a genetically-encoded anchoring disruptor by fusing PDE4D3 residues 1-20 comprising the 4D3 peptide (Fig. 6A) via a flexible linker $($ ELAAK $\times 3$ ) to the $\mathrm{N}$-terminus of mCherry (Fig. 9A). PDE4D3 binding to $\mathrm{mAKAP}$ is enhanced by PKA phosphor- 
A

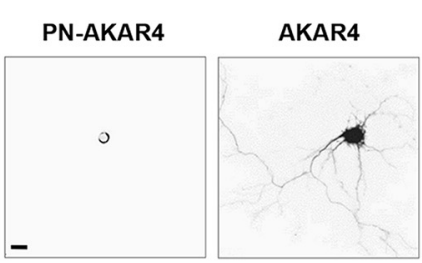

B
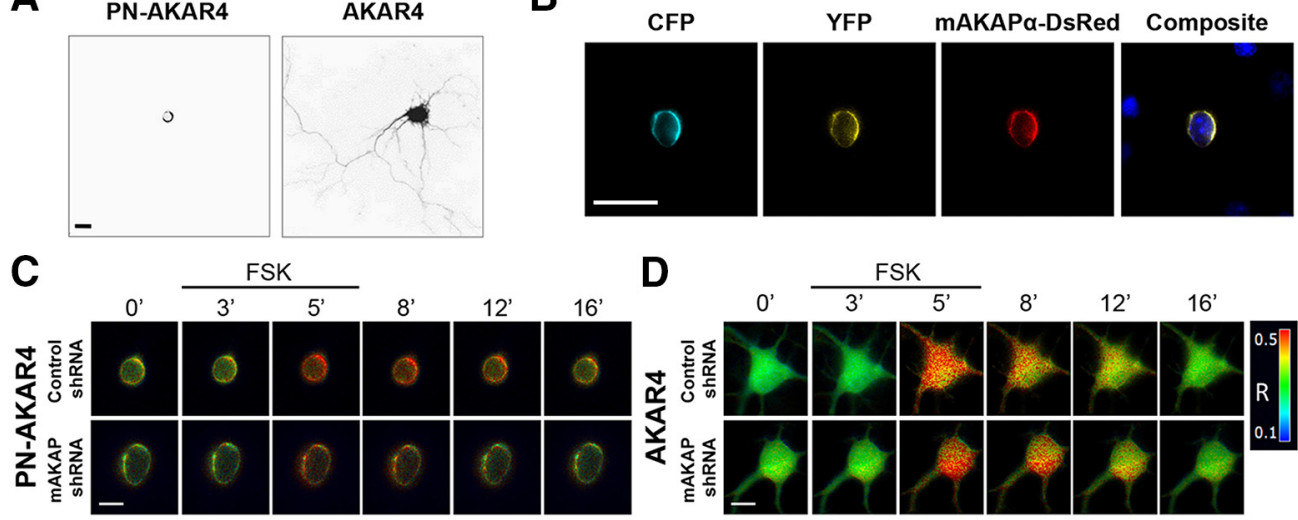

E

Amplitude $\left(\mathrm{R} / \mathrm{R}_{\mathbf{0}}\right)$

$t_{1 / 2}(s)$
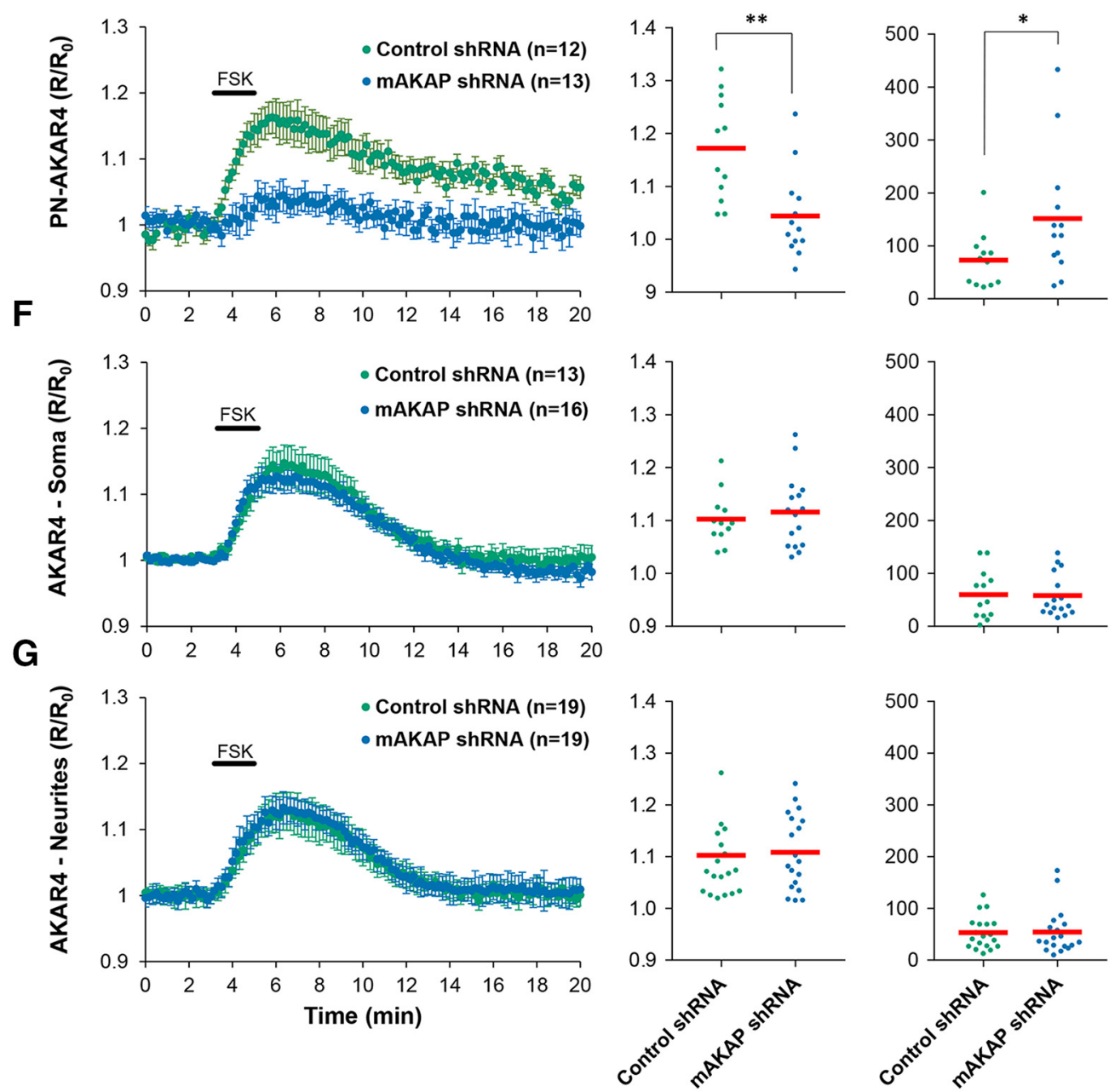

Figure 3. PN-AKAR4 is an mAKAP $\alpha$-dependent PKA sensor in hippocampal neurons. $\boldsymbol{A}$, Grayscale CFP images of PN-AKAR4 and AKAR4 sensors in neurons. Scale bar, $100 \mu \mathrm{m} . \boldsymbol{B}$, Colocalization of mAKAP $\alpha$-DsRed and PN-AKAR4 (CFP and YFP channels). Scale bar, $10 \mu \mathrm{m}$. C, D, Pseudocolored images showing FRET ratio $R$ (net FRET $\div$ background-subtracted donor signal) for representative tracings for $\boldsymbol{E}-\boldsymbol{G}$, The AKAR4 soma and neurite measurements were obtained from the same images by measuring the respective areas of the cells. Color hue and not intensity is indicative of $R$, as shown on the color scale. $E-G$, Neurons were infected with adenovirus for PN-AKAR4 or AKAR4 and for mAKAP or control shRNA and stimulated with $10 \mu \mathrm{m}$ FSK for 2 min (horizontal bars). Average tracings $\left(R / R_{0} \pm \mathrm{SEM}\right)$ and the peak amplitude and half-time of signal decay $\left(t_{1 / 2}\right)$ for individual tracings are shown; red bars indicate mean. ${ }^{*} p \leq 0.05,{ }^{* *} p \leq 0.01$.

ylation of residue PDE4D3 Ser-13 that can be mimicked by Ser to Glu substitution (Carlisle Michel et al., 2004). To increase the affinity and potency of the anchoring disruptor, the mCherry fusion peptide included this Ser ${ }^{13} \mathrm{Glu}$ missense mutation, "4D3(E)". Consistent with our hypothesis that displacement of $\mathrm{PDE} 4 \mathrm{D} 3$ from $\mathrm{mAKAP} \alpha$ signalosomes would increase cAMP persistence and PKA activity, expression of 4D3(E)-mCherry in hippocampal neurons increased baseline FRET signal 1.5-fold compared with mCherry control (Fig. 9C,E), and potentiated the PN-AKAR4 FRET response to FSK pulse 1.9-fold in amplitude and 2.8-fold in duration (Figs. 9C,E,F). Importantly, even though the $4 \mathrm{D} 3(\mathrm{E})$-mCherry peptide was diffusely expressed 
A mCherry-AC-nesprin

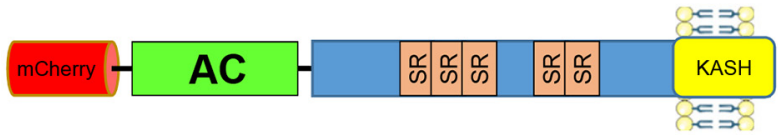

B
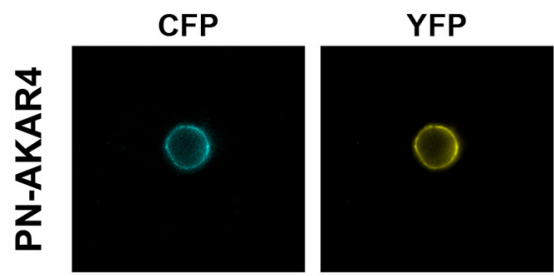

mCherry-AC
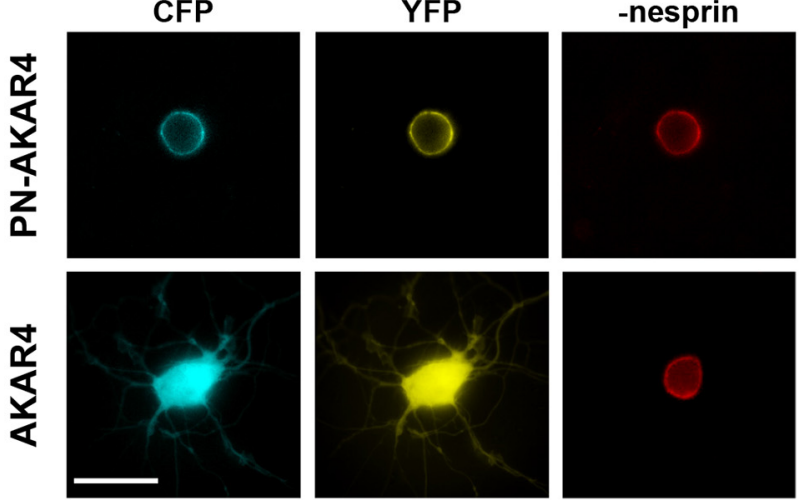

Composite

C
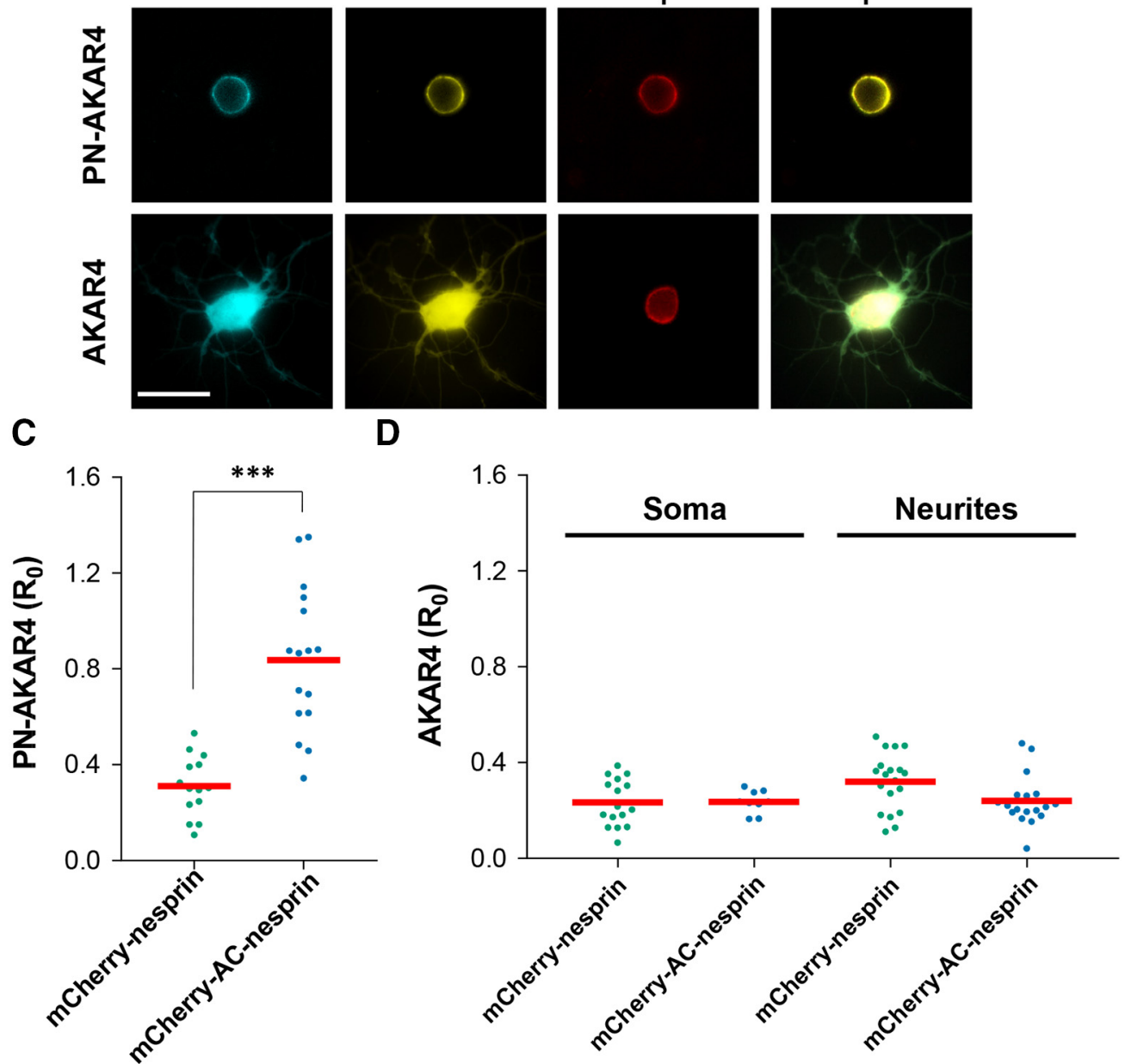

Figure 4. CAMP can be elevated selectively at the nuclear envelope. $A$, In mCherry-AC-nesprin, $m$ Cherry and the constitutively active catalytic domain of ADCY 10 are fused to the $N$-terminus of full-length nesprin-1 $\alpha . B$, Images of hippocampal neurons expressing mCherry-AC-nesprin and PN-AKAR4 or AKAR4 (CFP and YFP channels). Scale bar, $20 \mu \mathrm{m} . \mathbf{C}, \boldsymbol{D}$, Baseline FRET ratio $\left(R_{0}=\right.$ net FRET $\div$ Donor $)$ for PN-AKAR4 $(n=14,16)$ and AKAR4 $(n=9-19)$ was measured using hippocampal neurons expressing mCherry-AC-nesprin or control mCherry-nesprin; red bars indicate mean $* * * 0.001$.

A

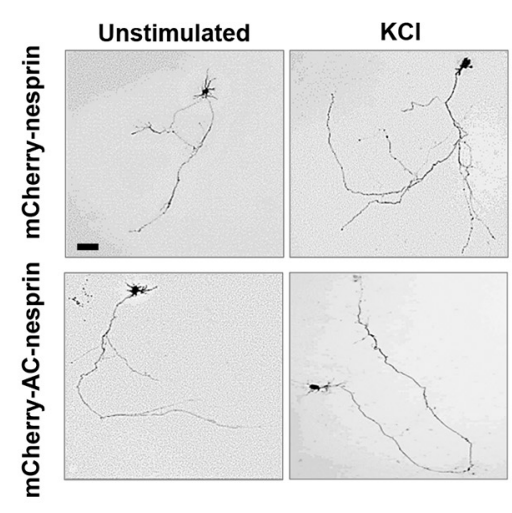

B

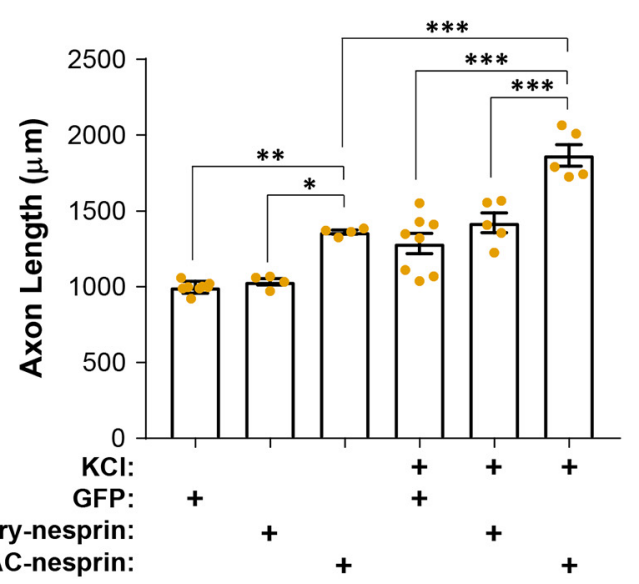

Figure 5. Elevated perinuclear cAMP is sufficient to promote neurite outgrowth. $A$, Hippocampal neurons expressing GFP and either mCherry-nesprin control or mCherry-AC-nesprin were cultured in defined media in the absence or presence of KCl for $2 \mathrm{~d}$. Grayscale images of GFP fluorescence are shown. Scale bar, $100 \mu \mathrm{m}$. $\boldsymbol{B}$, The length of the longest neurite was measured. $n=4-8$ independent neuronal cultures. ${ }^{*} p \leq 0.05,{ }^{* *} p \leq 0.01,{ }^{* * *} p \leq 0.001$. 


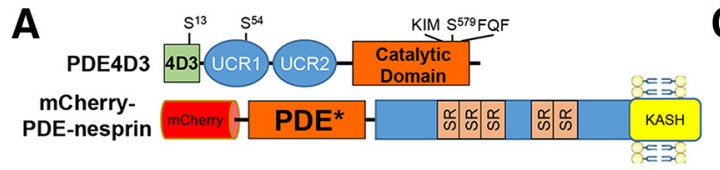

B
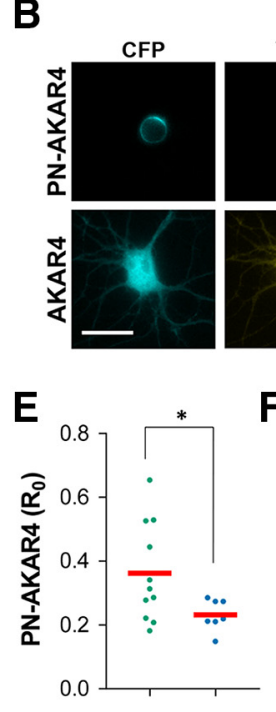

$\mathbf{F}$

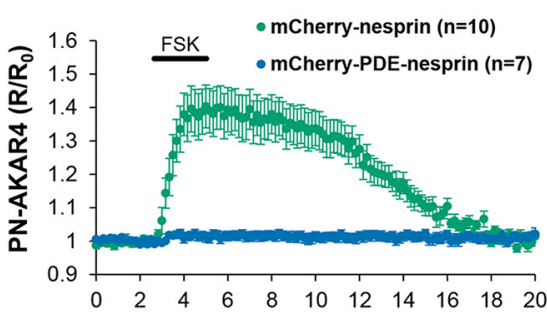

H
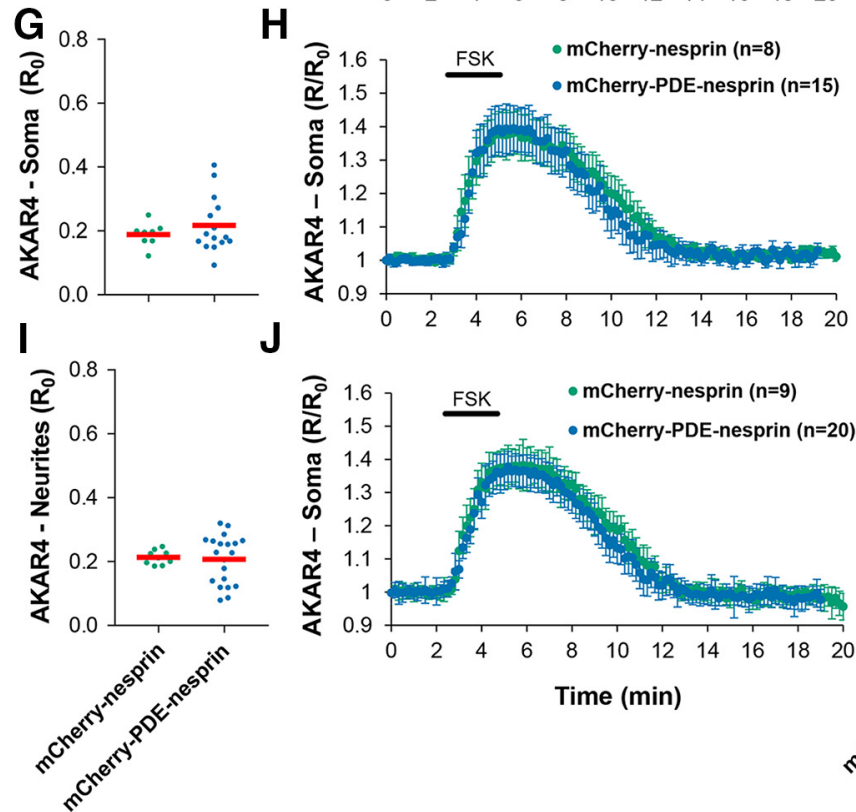
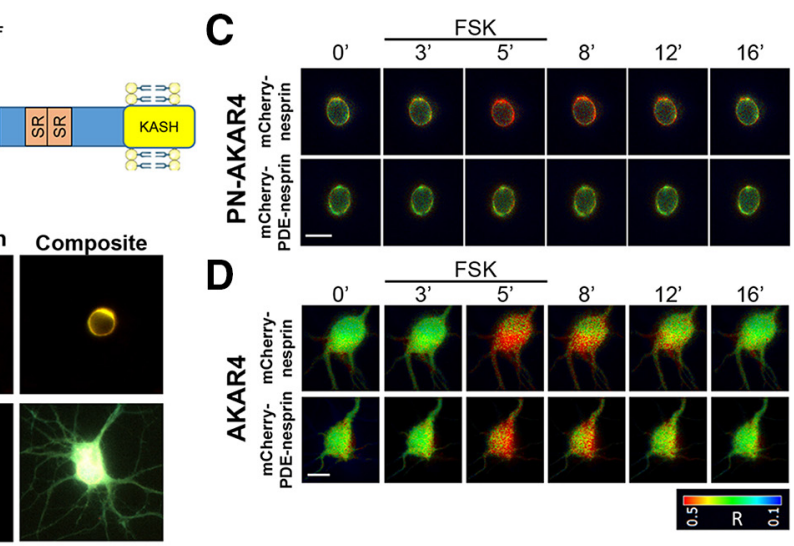

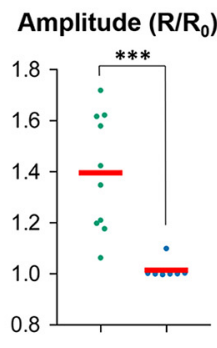

$t_{1 / 2}(s)$
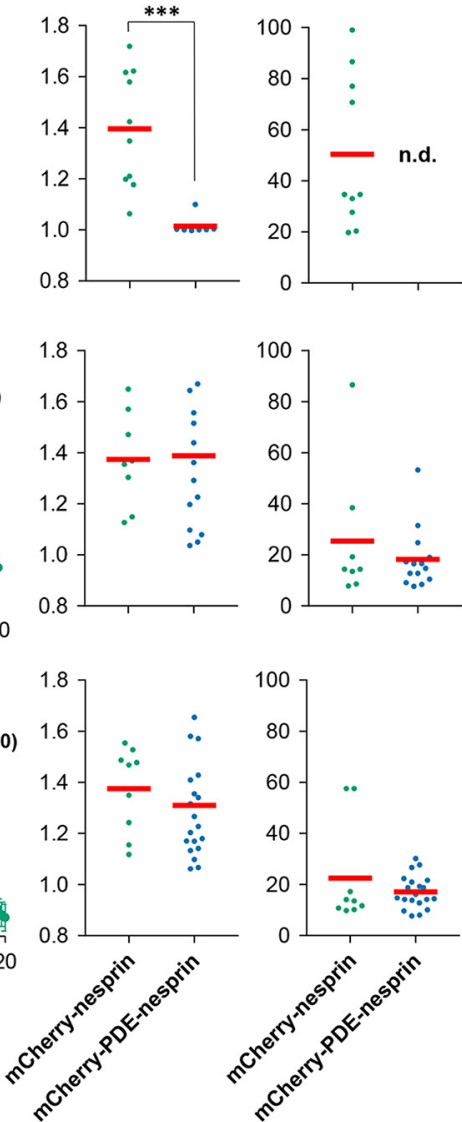

Figure 6. CAMP can be selectively depleted at the nuclear envelope. $A$, PDE4D3 contains a unique N-terminal " $4 D 3$ " peptide, two upstream conserved region (UCR1 and UCR2) and a C-terminal catalytic domain (Carlisle Michel et al., 2004). PKA phosphorylates Ser-13 and Ser-54 increasing mAKAP binding and catalytic activity, respectively. ERK binds the KIM and FQF motifs and phosphorylates Ser-579, inhibiting activity (MacKenzie et al., 2000). In mCherry-PDE-nesprin, mCherry and a mutant PDE4D catalytic domain (K455/K456/S579/F597/0598/ F599 all to alanine, PDE*) are fused to the N-terminus of full-length nesprin-1 $\alpha$. B. Images of hippocampal neurons expressing mCherry-PDE-nesprin and PN-AKAR4 or AKAR4 (CFP and YFP channels). Scale bar, $20 \mu \mathrm{m}$. C, $\boldsymbol{D}$, Pseudocolored images showing FRET ratio $R$ (net FRET $\div$ background-subtracted donor signal) for representative tracings for $\boldsymbol{F}, \boldsymbol{H}$, and $\boldsymbol{J}$. The AKAR4 soma and neurite measurements were obtained from the same images by measuring the respective areas of the cells. Color hue and not intensity is indicative of $R$, as shown on the color scale. $E, G, I$, Baseline FRET ratio ( $R_{0}=$ net FRET $\div$ Donor) was measured using neurons expressing PN-AKAR4 or AKAR4 and mCherry-PDE-nesprin or control mCherry-nesprin; red bars indicate mean. $\boldsymbol{F}, \boldsymbol{H}, J$, FRET tracings were obtained following stimulation with $10 \mu \mathrm{M}$ FSK for 2 min (horizontal bars). Average tracings $\left(R / R_{0} \pm S E M\right)$ and the peak amplitude and half-time of signal decay $\left(t_{1 / 2}\right)$ for individual tracings are shown. ${ }^{*} p \leq 0.05,{ }^{* * *} p \leq 0.001$.

throughout the cell (Fig. 9B), it had no significant effect on FRET signals obtained with the parent AKAR4 in the hippocampal neuron soma or neurites (Fig. 9D, G-J), thereby demonstrating the selectivity of 4D3(E)-mediated displacement of PDE4D3 from perinuclear mAKAP $\alpha /$ nesprin- $1 \alpha$ signalosomes.

Turning to axon growth, similar to the effects of mCherryAC-nesprin increasing cAMP/PKA at the perinuclear compart- ment, 4D3(E)-mCherry peptide expression increased axon extension $\sim 35 \%$ in hippocampal neurons (Fig. 10A,B) and $\sim 40 \%$ in RGCs as well (Fig. 10C,D). These results indicate that $\mathrm{PDE} 4 \mathrm{D} 3$ at perinuclear $\mathrm{mAKAP} \alpha$ in neurons regulates cAMP signals and PKA activity in that compartment and demonstrates conservation of PDE4D3 function between two $\operatorname{mAKAP} \alpha$ expressing CNS neuronal cell types. 

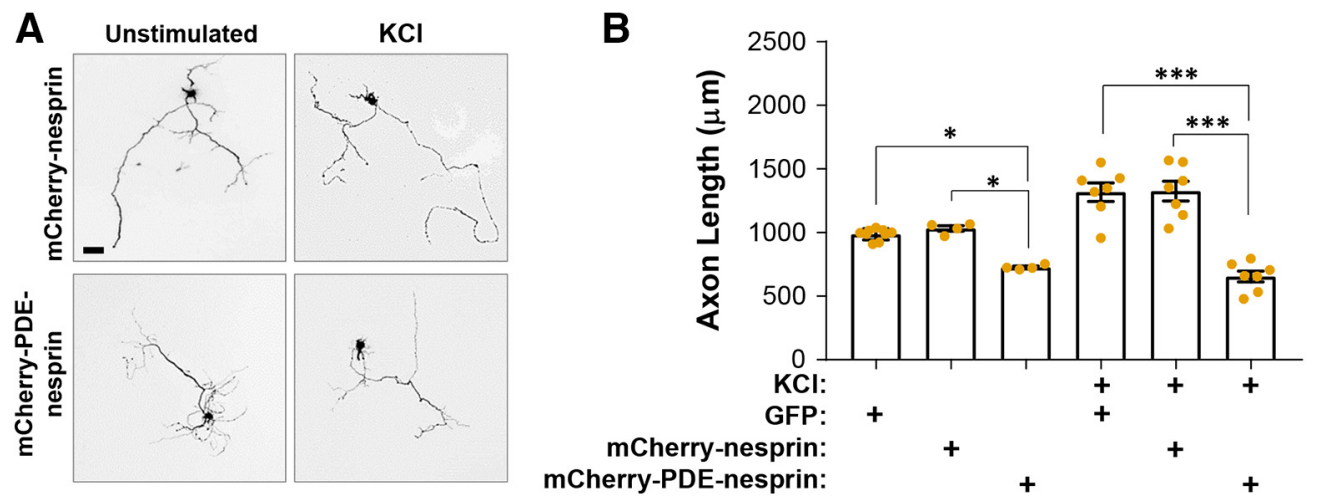

Figure 7. Perinuclear cAMP is required for neurite outgrowth in hippocampal neurons. $A$, Neurons expressing GFP and either mCherry-nesprin control or mCherry-PDE-nesprin were cultured in defined media in the absence or presence of $\mathrm{KCl}$ for $2 \mathrm{~d}$. Grayscale images of GFP fluorescence are shown. Scale bar, $100 \mu \mathrm{m}$. $\boldsymbol{B}$, The length of the longest neurite was measured. $n=4-10$ independent neuronal cultures. ${ }^{*} p \leq 0.05,{ }^{* *} p \leq 0.001$.

A
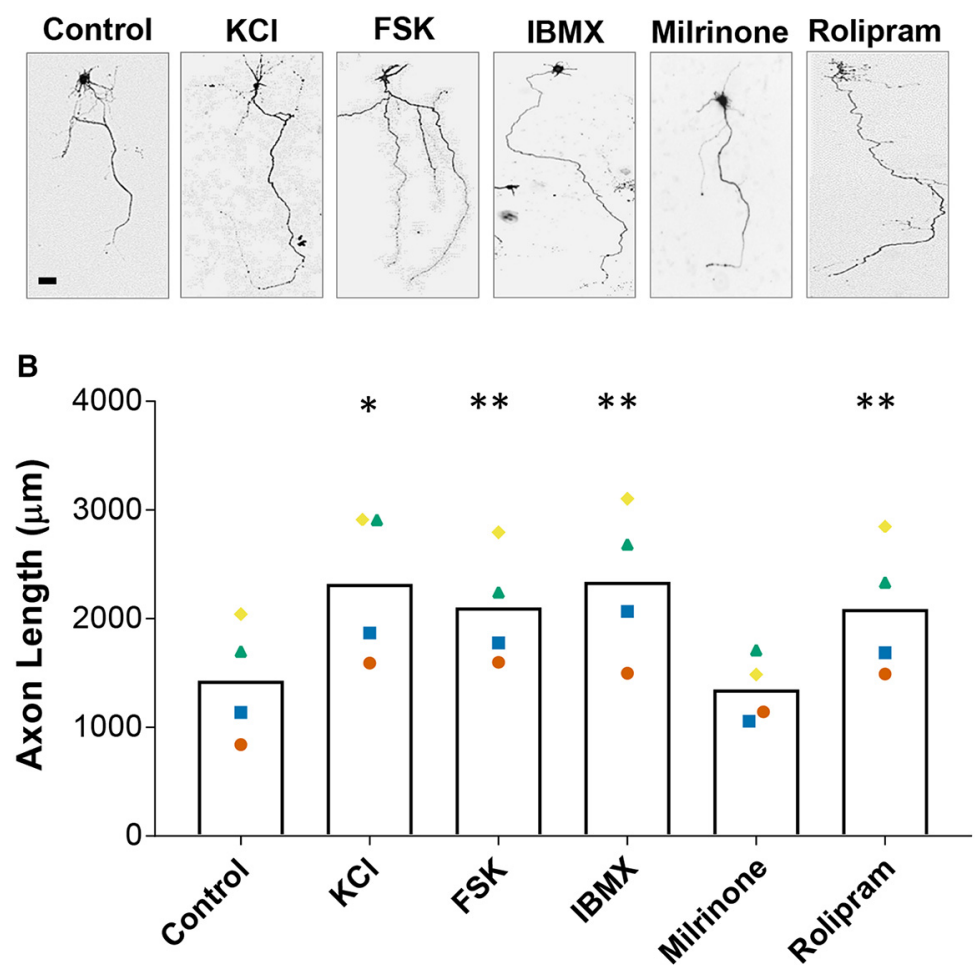

Figure 8. Pharmacological induction of neurite outgrowth. $A$, Hippocampal neurons transfected with a GFP expression plasmid were treated with $40 \mathrm{~mm} \mathrm{KCl}, 10 \mu \mathrm{m}$ FSK, $100 \mu \mathrm{m} \mathrm{IBMX,} 20 \mu \mathrm{m}$ milrinone, or $10 \mu \mathrm{m}$ rolipram for $2 \mathrm{~d}$. Grayscale images of GFP fluorescence are shown. Scale bar, $100 \mu \mathrm{m}$. $\boldsymbol{B}$, Mean length of the longest neurite. Colors represent paired data for four independent experiments. ${ }^{*} p$ value versus control. ${ }^{*} p \leq 0.05,{ }^{* *} p \leq 0.01$.

\section{RGC survival after optic nerve crush is enhanced by PDE4D3 anchoring disruption}

Previously we showed that in addition to axon growth in vitro, expression of the mAKAP $\alpha$ scaffold is required for the beneficial effects of exogenous neurotrophic factor and cAMP-analogs for the survival of RGC neurons following optic nerve crush injury (Wang et al., 2015). Intravitreal AAV2 preferentially transduces RGCs, allowing cell-type-selective gene delivery and peptide expression (Park et al., 2008). To test in vivo whether cAMP specifically localized at $\operatorname{mAKAP} \alpha$ signalosomes functions as an autonomous signaling compartment promoting neuroprotection or axon regeneration, we injected mice intravitreally with AAV2 for 4D3(E)-mCherry peptide or mCherry control (Fig.
11A). Anterograde labeling of RGC axons $24 \mathrm{~h}$ before euthanasia revealed that PDE4D3 displacement did not promote axonal regeneration within 2 weeks after optic nerve crush (data not shown). However, flat mount retinal staining for the RGC-specific marker RBPMS (Rodriguez et al., 2014) revealed that RGC survival was increased $30-50 \%$ with the $4 \mathrm{D} 3$ displacing peptide compared with control AAV2.mCherry alone, whether compared with the control uncrushed contralateral eye (Fig. $11 B, C$ ) or in an independent experiment with a different masked investigator and quantified as absolute RGC cell density (Fig. $11 D, E$ ). These results show that PDE4D3-anchoring disruption provides a method for enhancing physiologically relevant cAMP signaling in the $\mathrm{mAKAP} \alpha$ signaling compartment that promotes neuronal survival after injury.

\section{Discussion}

Using new molecular tools to induce or suppress cAMP levels at the mAKAP $\alpha$ scaffold, we demonstrate that perinuclear signalosomes constitutes a unique cAMP signaling compartment within neurons that regulates neuronal survival in vivo and axon growth in vitro. Despite obvious PKA activity in the soma, suppression of cAMP levels exclusively at mAKAP $\alpha$ signalosomes using mCherry-PDE-nesprin prevented baseline and depolarization-induced axon growth. Conversely, elevating cAMP levels using mCherry-ACnesprin was sufficient to induce axon growth. The use of PKA activity reporters not only validated the new molecular tools as specific for perinuclear signaling, but also provide evidence for cAMP compartmentation. Live cell imaging using nesprin- $1 \alpha$ localized and parent AKAR4 FRET biosensors showed that even though $\mathrm{mAKAP} \alpha$ signalosomes are localized to the nuclear envelope where there should be no physical barrier to cAMP diffusion, local perinuclear production of cAMP sufficient to alter cellular phenotype did not result in a detectable increase in PKA activity in the soma or neurites. These results support the model in which 
A

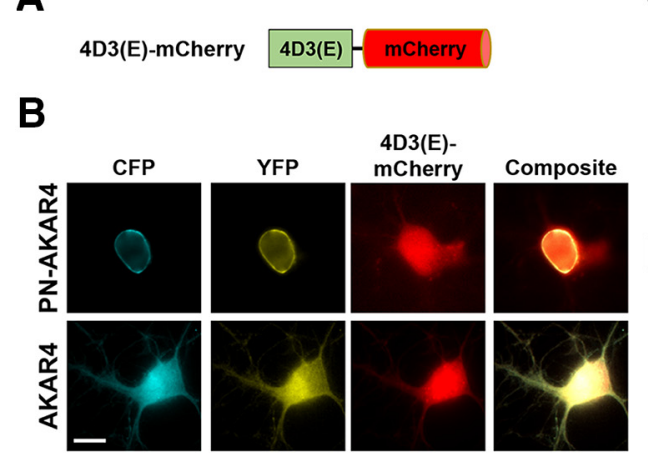

E

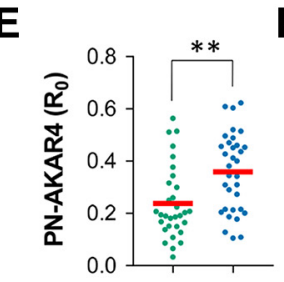

G

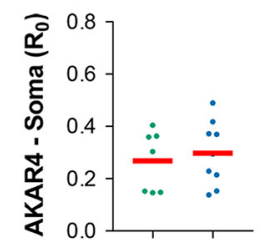

I

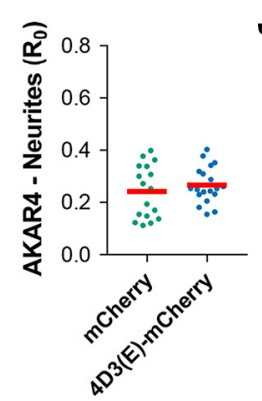

$\mathbf{F}$
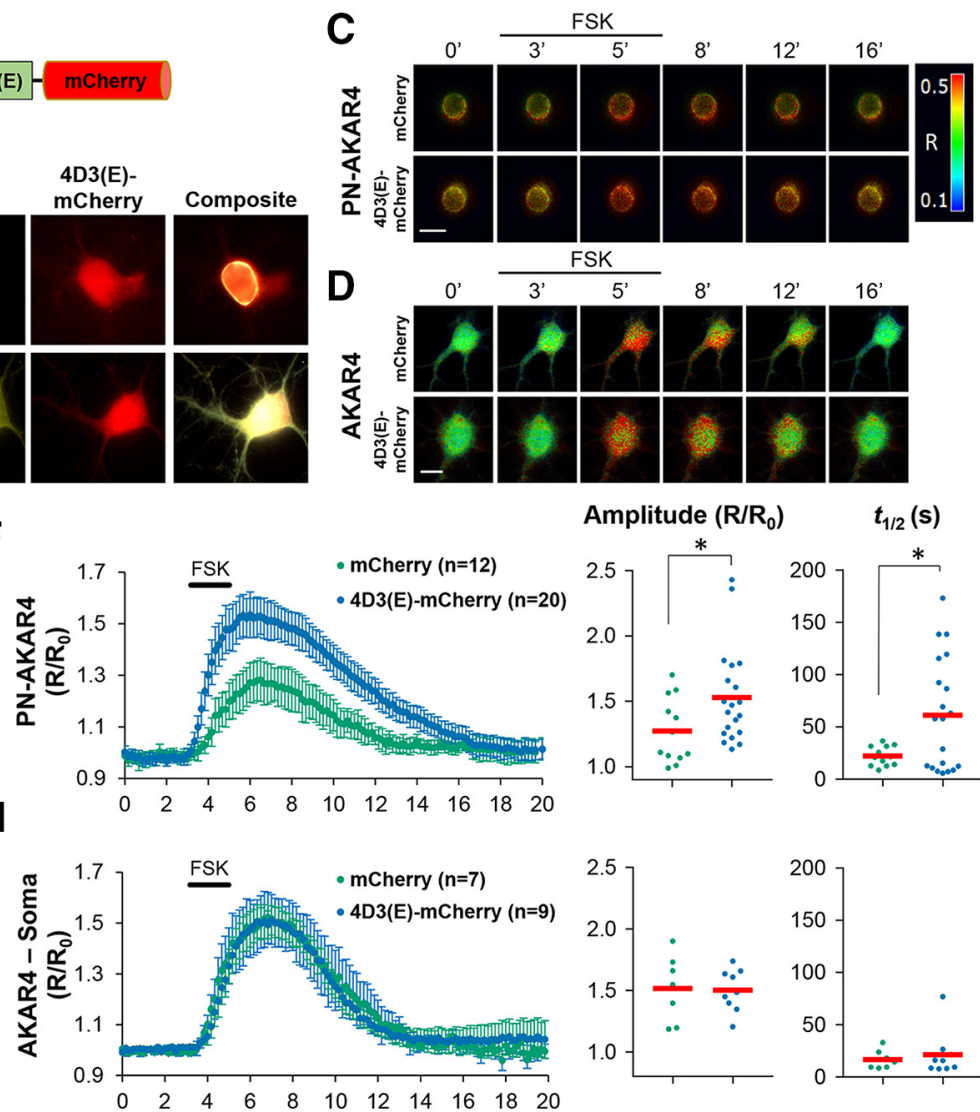

Amplitude $\left(\mathrm{R} / \mathrm{R}_{0}\right)$
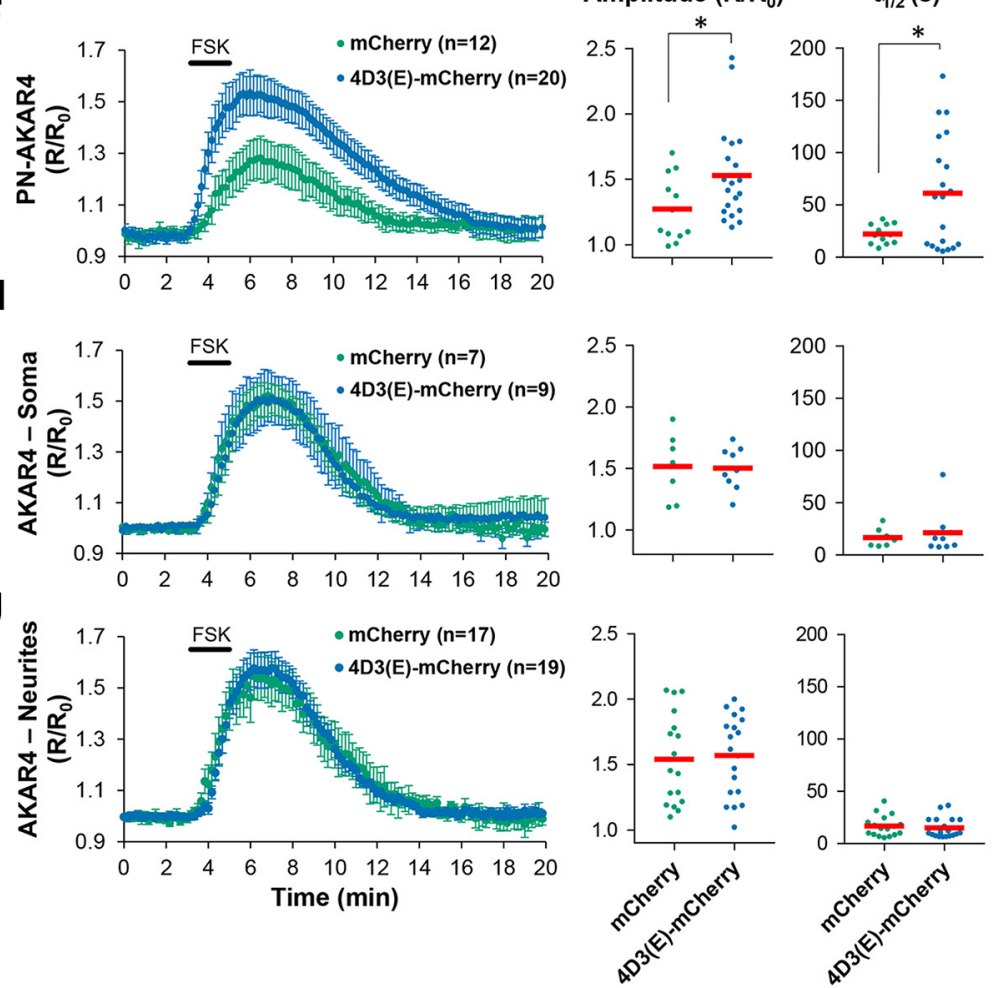

Figure 9. Displacement of PDE4D3 from mAKAP $\alpha$ increases perinuclear CAMP. $A, 4 D 3(E)$-mCherry includes the PDE4D3 isoform-specific N-terminal peptide with a Ser13Glu substitution in fusion to $\mathrm{mCherry}$. $\boldsymbol{B}$, Images of hippocampal neurons expressing the diffusely localized 4D3(E)-mCherry polypeptide and PN-AKAR4 or AKAR4 (CFP and YFP channels). Scale bar, $20 \mu \mathrm{m}$. C, D, Pseudocolored images showing FRET ratio $R$ (net FRET $\div$ background-subtracted donor signal) for representative tracings for $\boldsymbol{F}, \boldsymbol{H}$, and $\boldsymbol{J}$. The AKAR4 soma and neurite measurements were obtained from the same images by measuring the respective areas of the cells. Color hue and not intensity is indicative of $R$, as shown on the color scale. $E, G, I$, Baseline FRET ratio ( $R_{0}=$ net FRET $\div$ Donor) was measured using hippocampal neurons expressing PN-AKAR4 or AKAR4 and 4D3(E)-mCherry or control mCherry; red bars indicate mean. $\boldsymbol{F}, \boldsymbol{H}, J$, Tracings were obtained following stimulation for 2 min with $10 \mu \mathrm{m}$ forskolin (bar on graphs showing average $R / R_{0} \pm \mathrm{SEM}$ ). Peak amplitude and half-time of signal decay $\left(t_{1 / 2}\right)$ for individual tracings are shown on right. ${ }^{*} p \leq 0.05$, ${ }^{* *} p \leq 0.01$.

PKA action is spatially restricted by AKAP signalosomes that autonomously control local cAMP fluxes (Langeberg and Scott, 2015). This spatial specificity is critical given the multiplicity of cellular processes regulated by cAMP and PKA, including various steps in the formation of neural connectivity in which cAMP can play opposing roles (Averaimo and Nicol, 2014; Wild and Dell'Acqua, 2018).

Our results pertain to a "perinuclear cAMP compartment" containing $\mathrm{mAKAP} \alpha$, although not excluding the possibility that there are additional cAMP compartments near the nuclear envelope. However, given that PN-AKAR4 is $\operatorname{mAKAP} \alpha$-dependent in neurons, we suggest that the perinuclear compartment defined herein is limited to the immediate (nanometer) vicinity around nesprin- $1 \alpha$ at the nuclear envelope. This interpretation is consistent with evidence that PKA-AKAP signalosomes form discrete "nanocompartments," such that PKA only phosphorylates substrates physically associated with an AKAP scaffold, independently of targets that may be associated with the same organelle (Smith et al., 2017). Our results show that PDE4D3 binding to $\operatorname{mAKAP} \alpha$ tightly controls this cAMP compartment. Type $4 \mathrm{PDE}$ is a major source of cAMP degrading activity in neurons (Averaimo and Nicol, 2014; Gorshkov et al., 2017). PDE4 isoforms are distinguished by their individual N-terminal peptides that target them to different intracellular locations (Klussmann, 2016), and mAKAP $\alpha$ binds only type 4D3 PDE through its N-terminal peptide (Dodge et al., 2001). Displacement of individual signaling enzymes from signalosomes using anchoring disruptor peptides is an approach that allows the selective targeting of signalosome function without affecting global cellular signaling like classical pharmacologic inhibition (Klussmann, 2016). PDE4D3 displacement using 4D3(E)-mCherry specifically enhanced PN-AKAR4 signal and allowed the study of $\mathrm{mAKAP} \alpha$ signalosome function. 
A

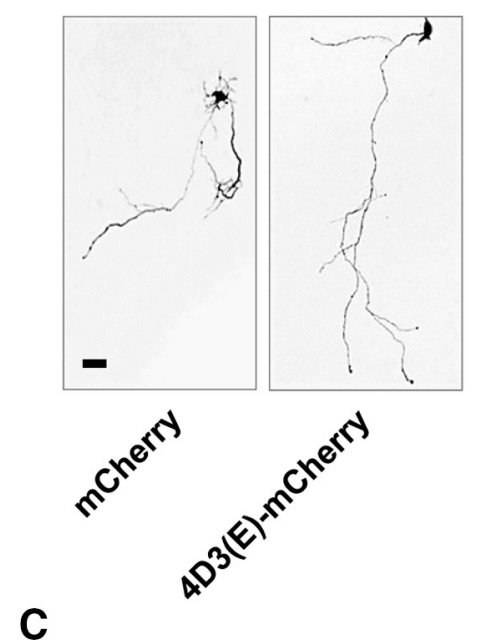

C

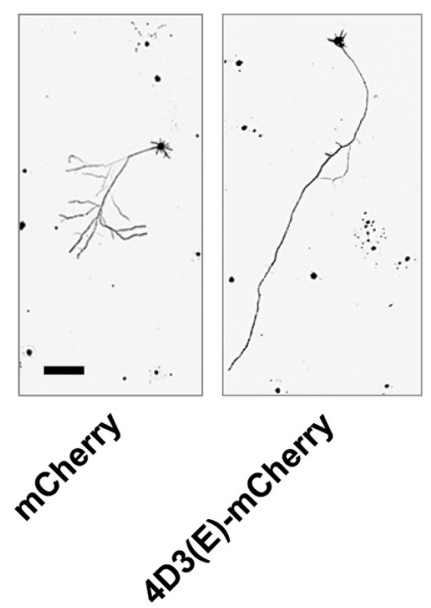

B

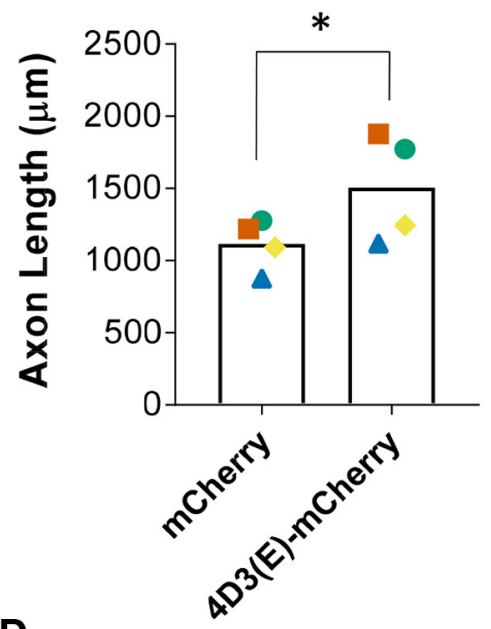

D

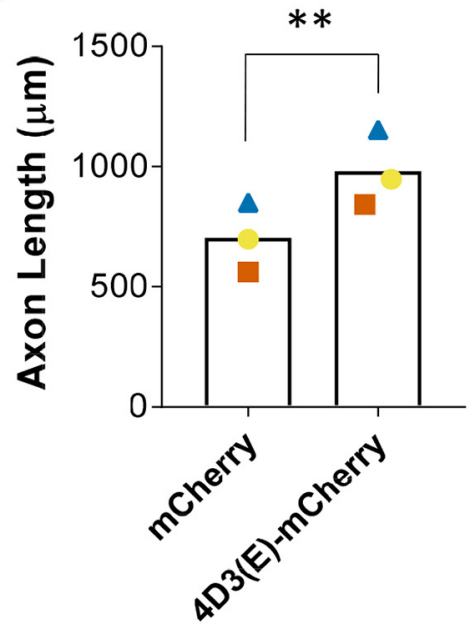

Figure 10. Displacement of PDE4D3 from mAKAP $\alpha$ promotes hippocampal and RGC neurite extension. $A$, Grayscale images of $m$ Cherry fluorescence for hippocampal neurons transfected with mCherry or 4D3(E)-mCherry expression plasmids and cultured for $2 \mathrm{~d}$ in defined media. Scale bar, $100 \mu \mathrm{m}$. $\boldsymbol{B}$, Mean lengths of the longest neurite are shown for four independent experiments (different colors). $\boldsymbol{C}, \boldsymbol{D}$, Same as $\boldsymbol{A}, \boldsymbol{B}$, except for RGCs. ${ }^{*} p \leq 0.05,{ }^{* *} p \leq 0.01$.

We have previously published that RGC-specific mAKAP $\alpha$ gene deletion blocked the neuroprotective effects of CPT-cAMP and BDNF after optic nerve crush (Wang et al., 2015). Whether the roles of mAKAP $\alpha$ in axon extension and neuroprotection involve similar downstream effectors, however, has remained unclear. To test the role of perinuclear cAMP in vivo, we used the anchoring disruptor peptide approach because mCherry-ACnesprin expression in vivo in a localized fashion is challenging because of the saturability of nesprin- $1 \alpha$ localization. Notably, enhanced cAMP signaling at mAKAP $\alpha$ by PDE4D3 displacement increased RGC survival after optic nerve injury. We found that AAV-mediated 4D3(E)-mCherry expression promoted neuroprotection as effectively as intravitreal injection of CPT-cAMP (Wang et al., 2015), although we did not directly compare these in the current study. It should be noted that in these experiments 4D3(E)-mCherry did not promote axon regeneration, consistent with previous findings that cAMP signaling alone does not promote axon regeneration (Kurimoto et al., 2010). Together, our data show that specific anchoring disruption of a relevant single PDE isoform is sufficient to promote localized PKA signaling and alter cellular phenotype in a manner consistent with the function of the corresponding scaffold protein even in the absence of altered global PKA signaling.
cAMP-dependent signaling is relevant to formation of neuronal cell networks during development, as well as survival and axon regeneration in the adult after injury (Kurimoto et al., 2010; Averaimo and Nicol, 2014). The formation of neuronal connections involves multiple cAMP-dependent steps, including polarization of immature neurons, axon elongation and branching, axon target guidance, and pruning of inappropriate synapses (Zhang and Poo, 2001; Nicol et al., 2011; Cheng and Poo, 2012). For example, a cAMP compartment at plasma membrane lipid rafts is important for ephrin-A regulated axonal guidance and pruning (Averaimo et al., 2016). As these latter effects are associated with local growth cone cAMP elevation, it is unlikely that the mAKAP $\alpha /$ nesprin- $1 \alpha$ compartment is involved. Although $\operatorname{mAKAP} \alpha-$ associated signaling is unlikely to be relevant to all of the different steps in neuronal development, mAKAP $\alpha$ is poised because of its perinuclear location to regulate gene expression through the post-translational modification of transcription factors and histone deacetylases as well as other nuclear enzymes that might regulate specific and longer-term aspects of the axon growth program (Fig. 11F; Passariello et al., 2015; Dodge-Kafka et al., 2018). To begin testing this hypothesis, we screened for changes in both the expression and phosphorylation of several previously identified regenerationassociated transcription factors (STAT3, CREB, c-JUN) and a growth-associated protein (GAP43) in response to PDE4D3 anchoring disruption. We detected no differences for these proteins by Western blot (data not shown); thus, further research will be required to identify downstream effectors for perinuclear cAMP. It has been reported that elevated neuronal cAMP levels promotes TrkB receptor plasma membrane translocation, potentiating neurotrophic signaling (Meyer-Franke et al., 1998). To begin testing the relationship between perinuclear cAMP and BDNF signaling, we measured PN-AKAR4 responsiveness to acute BDNF application. In these experiments, BDNF did not affect PN-ARAR4 FRET activity (data not shown), supporting the hypothesis that perinuclear cAMP is upstream of BDNF signaling or comprises a parallel pathway.

Does signaling in the mAKAP $\alpha /$ nesprin- $1 \alpha$ compartment mediate the survival-and axon growth-promoting effects of neuronal activity? It is well established that signaling by cAMP, including that produced by soluble adenylyl cyclase and mediated by PKA, is required for activity-dependent axon growth (Goldberg et al., 2002b). Our results show that increasing local cAMP with mCherry-AC-nesprin and depolarizing neurons with $\mathrm{KCl}$ stimulation similarly promote neurite outgrowth while depleting perinuclear cAMP suppressed axon elongation and fully occluded $\mathrm{KCl}$ 's ability to promote outgrowth. $\mathrm{KCl}$ stimulation may also activate mAKAP $\alpha$-independent pathways, but KCl's depen- 
A

AAV2sc.4D3(E)

B

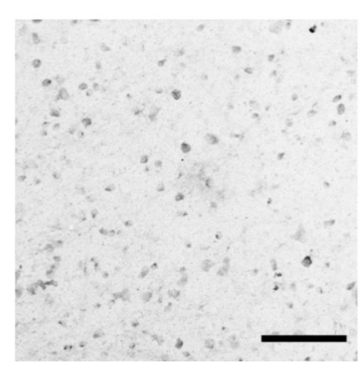

mCherry

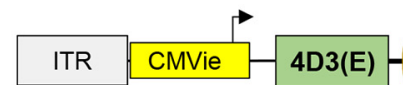

4D3(E)

mCherry

C

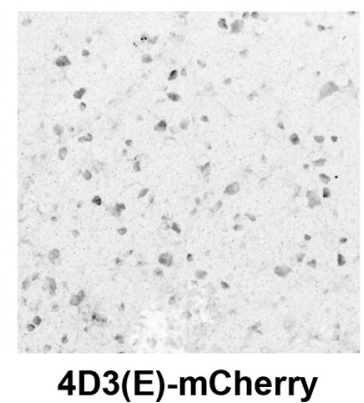

D
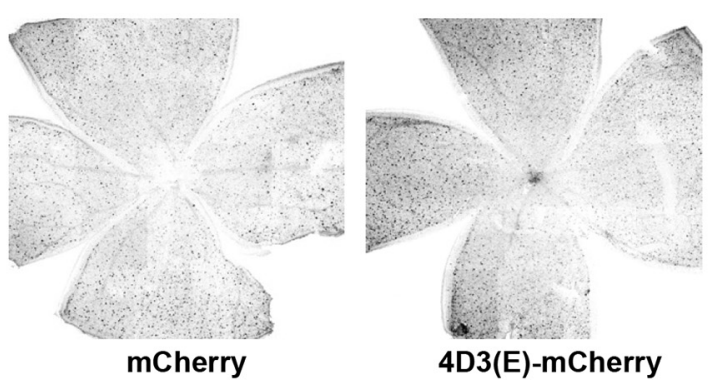

E
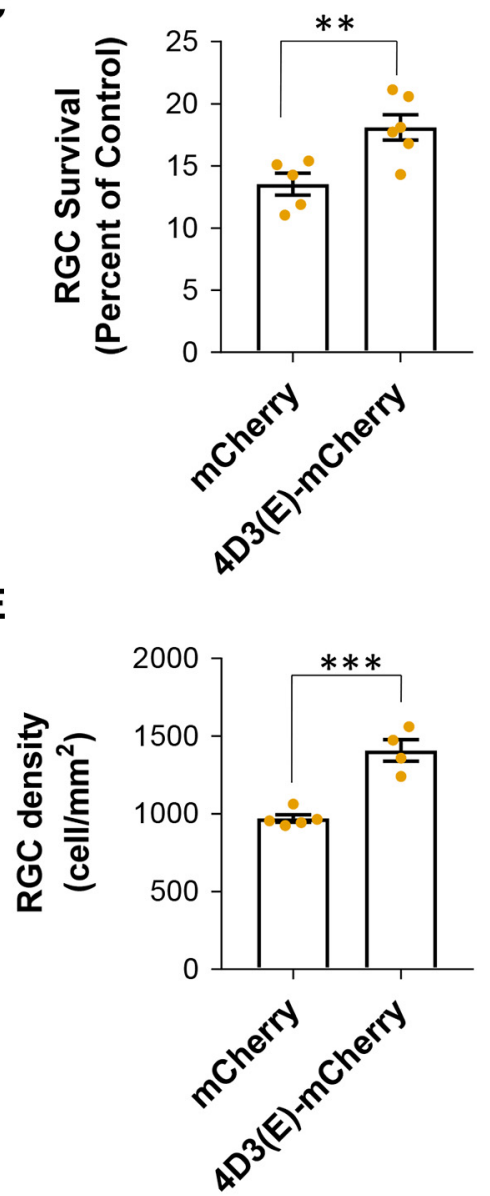

$\mathbf{F}$

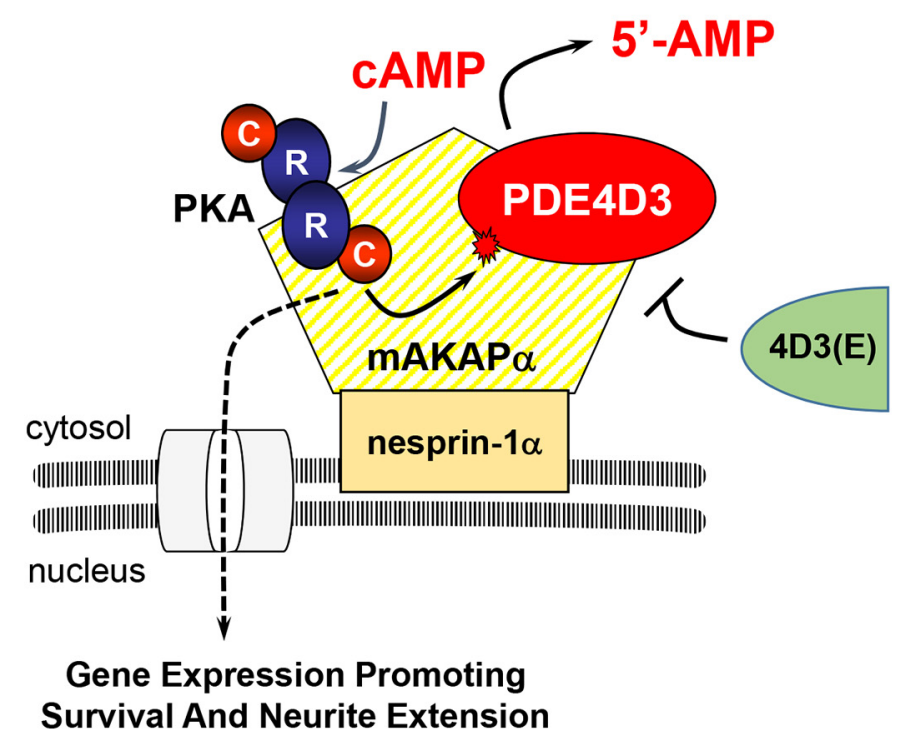

Figure 11. PDE4D3 anchoring disruption increases RGC survival after optic nerve crush. $\boldsymbol{A}, 4 \mathrm{DB}(\mathrm{E})$-mCherry was expressed in vivo using the gene therapy vector $\mathrm{AAV} 2 \mathrm{sc} .4 \mathrm{D} 3(\mathrm{E}) . \boldsymbol{B}, \mathrm{Retinas}$ isolated 2 weeks after optic nerve crush were stained for the RGC marker RBPMS (shown in grayscale). Scale bar, $100 \mu \mathrm{m}$. C, Quantification of RBPMS-stained cells showing increased RGC survival after AAV2.4D3(E) injection. $n=5,6$ mice. $\boldsymbol{D}, \boldsymbol{E}$, Same as in $\boldsymbol{B}$ and $\boldsymbol{C}$ except performed by a different investigator. $n=4,5$ mice. ${ }^{* *} p \leq 0.01,{ }^{* * *} p \leq 0.001$. $\boldsymbol{F}$, Model for regulation of perinuclear cAMP by mAKAP $\alpha$ signalosomes. mAKAP $\alpha$ binds the cAMP-specific, PKA-activated phosphodiesterase PDE4D3 that will oppose local PKA signaling in response to cAMP. The 4D3(E) peptide will displace PDE4D3 from mAKAP $\alpha$ potentiating local PKA signaling that promotes neuroprotection and neurite extension. 
dence on perinuclear cAMP revealed that the mAKAP $\alpha$ compartment is required for activity-dependent axon growth. The apparently independent effect of directly increasing perinuclear cAMP levels and $\mathrm{KCl}$ stimulation may simply reflect the quantitative ability of $\mathrm{KCl}$ to promote such elevations, because, for example, neurons rapidly desensitize to chronic $\mathrm{KCl}$ exposure. Axon damage induced by injury such as from increased intraocular pressure leads to enhanced RGC excitability that can temporarily delay degeneration (Risner et al., 2018). It has also been reported that intrinsically photosensitive retinal ganglion cells (ipRGCs) are more resistant to insult than other RGC subtypes because of their increased electrical excitability (Li et al., 2006; Daniel et al., 2018). In these examples, increased electrical activity correlates with enhanced neuroprotection, consistent with the enhanced RGC survival with PDE4D3 displacement in vivo. Still, whether all activity-induced cAMP signals associated with neuroprotection and outgrowth are mediated through $\mathrm{mAKAP} \alpha$ will require further study.

Cumulatively, our data suggest that $\operatorname{mAKAP} \alpha$ signalosomes selectively coordinate activity-dependent cAMP signaling that enables increased axonal growth in vitro and promotes neuroprotection after injury in vivo. These data demonstrate that the localization, kinetics, and phenotypic effects of this signaling are defined by PDE4D3 and PKA. Interestingly, increasing perinuclear cAMP was sufficient to promote neurite outgrowth in vitro, but not axonal regeneration in vivo. As our outgrowth assays were performed on perinatal neurons in culture, which exhibit enhanced growth potential relative to mature neurons (Goldberg et al., 2002a), the context in which mAKAP-dependent cAMP signaling is stimulated likely determines the ultimate phenotype. Thus, it may be necessary to reactivate developmental growth programs in combination with elevating perinuclear cAMP to achieve meaningful axon regeneration in mature neurons after injury. This notion is supported by findings that activity-induced cAMP elevation in injured RGCs potentiates the effects of growth-promoting manipulations such as mTOR activation (Kurimoto et al., 2010). How mTOR and other pathways interact with $\mathrm{mAKAP} \alpha$-orchestrated signaling will require further investigation, as will the identification of the relevant downstream effectors of mAKAP $\alpha$-associated cAMP signals. It is also possible that the inhibitory environment associated with crush injury precludes perinuclear cAMP-induced growth, such that promoting regeneration will require modulation of both intrinsic and extrinsic factors. Future work focused on how $\mathrm{mAKAP} \alpha$-mediated cAMP signaling differs in various disease models should provide clarity to this issue.

The potential significance of these results extends beyond a demonstration of mAKAP $\alpha$ signalosome compartmentation and function. RGC loss is a critical factor contributing to vision loss in many eye diseases, including in glaucoma, which is expected to affect $\sim 80$ million people worldwide by 2020 , of whom $\sim 10 \%$ are predicted to go blind. (Quigley and Broman, 2006) Given the great promise for AAV-based human ophthalmic therapies ( $\mathrm{Au}$ ricchio et al., 2017), AAV-based 4D3(E) anchoring disruptor expression constitutes an approach for the treatment of RGC neurodegenerative diseases that deserves further consideration.

\section{References}

Aglah C, Gordon T, Posse de Chaves EI (2008) cAMP promotes neurite outgrowth and extension through protein kinase A but independently of erk activation in cultured rat motoneurons. Neuropharmacology 55:817.

Auricchio A, Smith AJ, Ali RR (2017) The future looks brighter after 25 years of retinal gene therapy. Hum Gene Ther 28:982-987.
Averaimo S, Nicol X (2014) Intermingled cAMP, cGMP and calcium spatiotemporal dynamics in developing neuronal circuits. Front Cell Neurosci 8:376.

Averaimo S, Assali A, Ros O, Couvet S, Zagar Y, Genescu I, Rebsam A, Nicol $X$ (2016) A plasma membrane microdomain compartmentalizes ephrin-generated cAMP signals to prune developing retinal axon arbors. Nat Commun 7:12896.

Carlisle Michel JJ, Dodge KL, Wong W, Mayer NC, Langeberg LK, Scott JD (2004) PKA-phosphorylation of PDE4D3 facilitates recruitment of the mAKAP signalling complex. Biochem J 381:587-592.

Cheng PL, Poo MM (2012) Early events in axon/dendrite polarization. Annu Rev Neurosci 35:181-201.

Daniel S, Clark AF, McDowell CM (2018) Subtype-specific response of retinal ganglion cells to optic nerve crush. Cell Death Discov 4:7.

Depry C, Allen MD, Zhang J (2011) Visualization of PKA activity in plasma membrane microdomains. Mol Biosyst 7:52-58.

Dodge KL, Khouangsathiene S, Kapiloff MS, Mouton R, Hill EV, Houslay MD, Langeberg LK, Scott JD (2001) mAKAP assembles a protein kinase A/PDE4 phosphodiesterase cAMP signaling module. EMBO J 20:19211930.

Dodge-Kafka KL, Bauman A, Mayer N, Henson E, Heredia L, Ahn J, McAvoy T, Nairn AC, Kapiloff MS (2010) cAMP-stimulated protein phosphatase $2 \mathrm{~A}$ activity associated with muscle A kinase-anchoring protein (mAKAP) signaling complexes inhibits the phosphorylation and activity of the cAMP-specific phosphodiesterase PDE4D3. J Biol Chem 285: $11078-11086$.

Dodge-Kafka KL, Gildart M, Li J, Thakur H, Kapiloff MS (2018) Bidirectional regulation of HDAC5 by mAKAP $\beta$ signalosomes in cardiac myocytes. J Mol Cell Cardiol 118:13-25.

Goldberg JL, Klassen MP, Hua Y, Barres BA (2002a) Amacrine-signaled loss of intrinsic axon growth ability by retinal ganglion cells. Science 296 : $1860-1864$

Goldberg JL, Espinosa JS, Xu Y, Davidson N, Kovacs GT, Barres BA (2002b) Retinal ganglion cells do not extend axons by default: promotion by neurotrophic signaling and electrical activity. Neuron 33:689-702.

Gorshkov K, Mehta S, Ramamurthy S, Ronnett GV, Zhou FQ, Zhang J (2017) AKAP-mediated feedback control of cAMP gradients in developing hippocampal neurons. Nat Chem Biol 13:425-431.

Gros-Louis F, Dupré N, Dion P, Fox MA, Laurent S, Verreault S, Sanes JR, Bouchard JP, Rouleau GA (2007) Mutations in SYNE1 lead to a newly discovered form of autosomal recessive cerebellar ataxia. Nat Genet 39: $80-85$.

Kapiloff MS, Schillace RV, Westphal AM, Scott JD (1999) mAKAP: an A-kinase anchoring protein targeted to the nuclear membrane of differentiated myocytes. J Cell Sci 112:2725-2736.

Kapiloff MS, Piggott LA, Sadana R, Li J, Heredia LA, Henson E, Efendiev R, Dessauer CW (2009) An adenylyl cyclase-mAKAP $\beta$ signaling complex regulates cAMP levels in cardiac myocytes. J Biol Chem 284:2354023546.

Klussmann E (2016) Protein-protein interactions of PDE4 family members: functions, interactions and therapeutic value. Cell Signal 28:713-718.

Kurimoto T, Yin Y, Omura K, Gilbert HY, Kim D, Cen LP, Moko L, Kügler S, Benowitz LI (2010) Long-distance axon regeneration in the mature optic nerve: contributions of oncomodulin, cAMP, and pten gene deletion. J Neurosci 30:15654-15663.

Langeberg LK, Scott JD (2015) Signalling scaffolds and local organization of cellular behaviour. Nat Rev Mol Cell Biol 16:232-244.

Li J, Negro A, Lopez J, Bauman AL, Henson E, Dodge-Kafka K, Kapiloff MS (2010) The mAKAPbeta scaffold regulates cardiac myocyte hypertrophy via recruitment of activated calcineurin. J Mol Cell Cardiol 48:387-394.

Li RS, Chen BY, Tay DK, Chan HH, Pu ML, So KF (2006) Melanopsinexpressing retinal ganglion cells are more injury-resistant in a chronic ocular hypertension model. Invest Ophthalmol Vis Sci 47:2951-2958.

MacKenzie SJ, Baillie GS, McPhee I, Bolger GB, Houslay MD (2000) ERK2 mitogen-activated protein kinase binding, phosphorylation, and regulation of the PDE4D cAMP-specific phosphodiesterases: the involvement of $\mathrm{COOH}$-terminal docking sites and $\mathrm{NH} 2$-terminal UCR regions. J Biol Chem 275:16609-16617.

Marx SO, Reiken S, Hisamatsu Y, Gaburjakova M, Gaburjakova J, Yang YM, Rosemblit N, Marks AR (2001) Phosphorylation-dependent regulation of ryanodine receptors: a novel role for leucine/isoleucine zippers. J Cell Biol 153:699-708. 
Meyer-Franke A, Kaplan MR, Pfrieger FW, Barres BA (1995) Characterization of the signaling interactions that promote the survival and growth of developing retinal ganglion cells in culture. Neuron 15:805-819.

Meyer-Franke A, Wilkinson GA, Kruttgen A, Hu M, Munro E, Hanson MG Jr, Reichardt LF, Barres BA (1998) Depolarization and cAMP elevation rapidly recruit TrkB to the plasma membrane of CNS neurons. Neuron 21:681-693.

Michel JJ, Townley IK, Dodge-Kafka KL, Zhang F, Kapiloff MS, Scott JD (2005) Spatial restriction of PDK1 activation cascades by anchoring to mAKAP $\alpha$. Mol Cell 20:661-672.

Nicol X, Hong KP, Spitzer NC (2011) Spatial and temporal second messenger codes for growth cone turning. Proc Natl Acad Sci U S A 108: 13776-13781.

Pare GC, Easlick JL, Mislow JM, McNally EM, Kapiloff MS (2005a) Nesprin-1alpha contributes to the targeting of mAKAP to the cardiac myocyte nuclear envelope. Exp Cell Res 303:388-399.

Pare GC, Bauman AL, McHenry M, Michel JJ, Dodge-Kafka KL, Kapiloff MS (2005b) The mAKAP complex participates in the induction of cardiac myocyte hypertrophy by adrenergic receptor signaling. J Cell Sci 118:5637-5646.

Park KK, Liu K, Hu Y, Smith PD, Wang C, Cai B, Xu B, Connolly L, Kramvis I, Sahin M, He Z (2008) Promoting axon regeneration in the adult CNS by modulation of the PTEN/mTOR pathway. Science 322:963-966.

Passariello CL, Gayanilo M, Kritzer MD, Thakur H, Cozacov Z, Rusconi F, Wieczorek D, Sanders M, Li J, Kapiloff MS (2013) p90 ribosomal S6 kinase 3 contributes to cardiac insufficiency in alpha-tropomyosin Glu180Gly transgenic mice. Am J Physiol Heart Circ Physiol 305: H1010-H1019.

Passariello CL, Li J, Dodge-Kafka K, Kapiloff MS (2015) mAKAP-a master scaffold for cardiac remodeling. J Cardiovasc Pharmacol 65:218-225.

Quigley HA, Broman AT (2006) The number of people with glaucoma worldwide in 2010 and 2020. Br J Ophthalmol 90:262-267.

Razafsky DS, Ward CL, Kolb T, Hodzic D (2013) Developmental regulation of linkers of the nucleoskeleton to the cytoskeleton during mouse postnatal retinogenesis. Nucleus 4:399-409.

Risner ML, Pasini S, Cooper ML, Lambert WS, Calkins DJ (2018) Axogenic mechanism enhances retinal ganglion cell excitability during early progression in glaucoma. Proc Natl Acad Sci U S A 115:E2393-E2402.
Rodriguez AR, de Sevilla Müller LP, Brecha NC (2014) The RNA binding protein RBPMS is a selective marker of ganglion cells in the mammalian retina. J Comp Neurol 522:1411-1443.

Smith FD, Esseltine JL, Nygren PJ, Veesler D, Byrne DP, Vonderach M, Strashnov I, Eyers CE, Eyers PA, Langeberg LK, Scott JD (2017) Local protein kinase A action proceeds through intact holoenzymes. Science 356:1288-1293.

Stiles TL, Kapiloff MS, Goldberg JL (2014) The role of soluble adenylyl cyclase in neurite outgrowth. Biochim Biophys Acta 1842:2561-2568.

Terenzio M, Schiavo G, Fainzilber M (2017) Compartmentalized signaling in neurons: from cell biology to neuroscience. Neuron 96:667-679.

Vargas MA, Tirnauer JS, Glidden N, Kapiloff MS, Dodge-Kafka KL (2012) Myocyte enhancer factor 2 (MEF2) tethering to muscle selective A-kinase anchoring protein (mAKAP) is necessary for myogenic differentiation. Cell Signal 24:1496-1503.

Wang Y, Cameron EG, Li J, Stiles TL, Kritzer MD, Lodhavia R, Hertz J, Nguyen T, Kapiloff MS, Goldberg JL (2015) Muscle A-kinase anchoring protein-alpha is an injury-specific signaling scaffold required for neurotrophic- and cyclic adenosine monophosphate-mediated survival. EBioMedicine 2:1880-1887.

Wild AR, Dell'Acqua ML (2018) Potential for therapeutic targeting of AKAP signaling complexes in nervous system disorders. Pharmacol Ther 185:99-121.

Zhang LI, Poo MM (2001) Electrical activity and development of neural circuits. Nat Neurosci 4:1207-1214.

Zhang L, Malik S, Kelley GG, Kapiloff MS, Smrcka AV (2011) Phospholipase $\mathrm{C}$ epsilon scaffolds to muscle-specific $\mathrm{A}$ kinase anchoring protein $(\mathrm{mAKAP} \beta)$ and integrates multiple hypertrophic stimuli in cardiac myocytes. J Biol Chem 286:23012-23021.

Zhao Z, Xu P, Jie Z, Zuo Y, Yu B, Soong L, Sun J, Chen Y, Cai J (2014) $\gamma \delta$ T cells as a major source of IL-17 production during age-dependent RPE degeneration. Invest Ophthalmol Vis Sci 55:6580-6589.

Zhou C, Rao L, Shanahan CM, Zhang Q (2018) Nesprin-1/2: roles in nuclear envelope organisation, myogenesis and muscle disease. Biochem Soc Trans 46:311-320.

Zhou X, Herbst-Robinson KJ, Zhang J (2012) Visualizing dynamic activities of signaling enzymes using genetically encodable FRET-based biosensors from designs to applications. Methods Enzymol 504:317-340. 